\title{
Cosmology with inhomogeneous magnetic fields
}

\author{
John D. Barrow \\ DAMTP, Centre for Mathematical Sciences, Wilberforce Road, \\ Cambridge CB3 0WA, UK \\ Roy Maartens \\ Institute of Cosmology \& Gravitation, University of Portsmouth, \\ Portsmouth P01 2EG, UK \\ Christos G. Tsagas \\ Section of Astrophysics, Astronomy and Mechanics, Department of Physics, \\ Aristotle University of Thessaloniki, Thessaloniki 54124, Greece
}




\begin{abstract}
We review spacetime dynamics in the presence of large-scale electromagnetic fields and then consider the effects of the magnetic component on perturbations to a spatially homogeneous and isotropic universe. Using covariant techniques, we refine and extend earlier work and provide the magnetohydrodynamic equations that describe inhomogeneous magnetic cosmologies in full general relativity. Specialising this system to perturbed Friedmann-Robertson-Walker models, we examine the effects of the field on the expansion dynamics and on the growth of density inhomogeneities, including non-adiabatic modes. We look at scalar perturbations and obtain analytic solutions for their linear evolution in the radiation, dust and inflationary eras. In the dust case we also calculate the magnetic analogue of the Jeans length. We then consider the evolution of vector perturbations and find that the magnetic presence generally reduces the decay rate of these distortions. Finally, we examine the implications of magnetic fields for the evolution of cosmological gravitational waves.
\end{abstract}

PACS: 98.80.-k; 98.62.En; 98.65.Dx

Key words: Cosmology, Magnetic Fields, Large-Scale Structure

\title{
Contents
}

1 Introduction

$2 \quad \mathbf{1}+3$ covariant description 6

2.1 Local spacetime splitting 6

$\begin{array}{lll}2.2 & \text { Matter fields } & 7\end{array}$

$\begin{array}{lll}2.3 & \text { Covariant kinematics } & 7\end{array}$

3 Electromagnetic fields 8

3.1 Electric and magnetic components 9

$\begin{array}{llr}3.2 & \text { Maxwell's equations } & 9\end{array}$

$\begin{array}{lll}3.3 & \text { Conservation laws } & 10\end{array}$

$\begin{array}{lll}3.4 & \text { Ohm's law } & 11\end{array}$

4 Gravitational field 11

4.1 Local Ricci curvature $\quad 12$

4.2 Long-range Weyl curvature 13

$\begin{array}{lll}4.3 & \text { Spatial curvature } & 14\end{array}$

5 Non-linear cosmological magnetohydrodynamics 16

$\begin{array}{lll}5.1 & \text { Ideal MHD approximation } & 16\end{array}$

$\begin{array}{lll}5.2 \text { Magnetic evolution } & 17\end{array}$

$\begin{array}{ll}5.3 \text { Conservation laws } & 17\end{array}$

$\begin{array}{lll}5.4 & \text { Magnetic tension } & 18\end{array}$ 
$\begin{array}{llr}5.5 & \text { Kinematical evolution } & 19\end{array}$

$\begin{array}{lll}5.6 & \text { Spacetime curvature } & 20\end{array}$

$\begin{array}{lll}5.7 & \text { Evolution of inhomogeneities } & 21\end{array}$

6 Perturbed magnetic FRW cosmologies 22

$\begin{array}{lll}6.1 & \text { Background evolution } & 23\end{array}$

$\begin{array}{lll}6.2 & \text { Linear evolution } & 25\end{array}$

$\begin{array}{lll}6.3 & \text { Spatial inhomogeneities } & 27\end{array}$

7 Density perturbations 30

$\begin{array}{lll}7.1 & \text { Basic variables } & 30\end{array}$

$\begin{array}{lll}7.2 & \text { Linear equations } & 31\end{array}$

$\begin{array}{lll}7.3 & \text { Evolution in the radiation era } & 32\end{array}$

$\begin{array}{lll}7.4 & \text { Evolution in the dust era } & 36\end{array}$

$\begin{array}{lll}7.5 & \text { Evolution in false-vacuum environments } & 38\end{array}$

8 Isocurvature perturbations 40

8.1 Consistency condition 40

8.2 Evolution in the radiation era 41

$\begin{array}{lll}8.3 & \text { Evolution in the dust era } & 42\end{array}$

$9 \quad$ Entropy perturbations 43

9.1 Entropy perturbations in multi-component systems 43

9.2 Magnetically-induced entropy perturbations 43

10 Vector perturbations $\quad 44$

10.1 Basic variables 44

$\begin{array}{ll}10.2 \text { Linear equations } & 45\end{array}$

$\begin{array}{ll}10.3 \text { Evolution in the dust era } & 46\end{array}$

11 Gravitational waves 47

$\begin{array}{ll}11.1 \text { Isolating magnetised tensor modes } & 47\end{array}$

$\begin{array}{ll}11.2 \text { Covariant gravitational-wave energy density } & 49\end{array}$

$\begin{array}{ll}11.3 \text { Evolution equations } & 49\end{array}$

$\begin{array}{ll}11.4 \text { The zero-eigenvalue issue } & 50\end{array}$

12 Summary 52

A Commutation laws 53

A.1 Orthogonally projected gradients 53

A.2 Time derivatives $\quad 54$

B Notation $\quad 54$

$\begin{array}{ll}\text { References } & 55\end{array}$ 


\section{Introduction}

Magnetic fields are a widespread and significant component of the Universe [1]-[7]. The Milky Way and many other spiral galaxies possess magnetic fields with strengths of order a few micro Gauss, that are coherent over the plane of the galactic disc. Magnetic fields are also a common property of galaxy clusters, extending well beyond their core regions. The strengths of the ordered magnetic fields in the intracluster medium exceed those typically associated with the interstellar medium of the Milky Way, suggesting that galaxy formation, and even cluster dynamics, could be influenced by magnetic forces. Furthermore, reports of Faraday rotation in high-redshift, Lyman- $\alpha$ absorption systems suggest that dynamically significant magnetic fields could be present in protogalactic condensations.

Despite their widespread presence, however, the precise origin of any large-scale cosmological magnetic field is still a mystery, and its likelihood or necessity remains a subject of debate [8][12]. The alignment of the galactic fields, especially those seen in spiral galaxies, seems to support the dynamo idea [13]-[17. However, the galactic dynamo cannot work without an initial seed field [18]-20] and these seeds may require $B$-fields of primeval origin [21]-[44]. Deciding whether galactic and cluster magnetic fields are primordial relics or post-recombination artefacts is difficult because the strong amplification of the fields in these virialized systems has overwhelmed all traces of their earlier history. In contrast, possible magnetic imprints in the Cosmic Microwave Background (CMB), or a magnetic field in the intercluster space, can provide better insight into the early history because the presence of small-scale magnetic fields leaves small angular scale features in the CMB undamped [45,46], and leads to distinctive polarisation signatures [47. The idea of primordial magnetism is attractive because it can potentially explain all the large-scale $B$-fields seen in the universe today, especially those found in high-redshift protogalaxies. Early magnetogenesis is not problem-free however. Seed fields generated during, say, phase-transitions in the radiation era have typical coherence lengths that are very small and will destabilize the dynamo. On the other hand, fields which survived an epoch of inflation are typically too weak to sustain the dynamo. Although the literature contains several mechanisms of primordial magnetic amplification [48]-[57], with early reheating looking like a promising stage [58], the issue remains open. Any cosmological magnetic field must also be consistent with a number of astrophysical constraints. These come from primordial nucleosynthesis [59,60], and the high isotropy of the CMB radiation [61]-[80]. This constrains the current strength of a large-scale homogeneous $B$-field below $\sim 10^{-9} G$ [66] (see also $\S 11.4$ here), although tangled random fields on small scales could reach up to $\sim 10^{-6} G$.

Large-scale magnetic fields of $\mu G$ strength can affect the evolution of structure in the Universe, and studies of their effects have a long history. Anisotropic, spatially homogeneous, relativistic cosmologies permeated by large-scale magnetic fields have been analysed in [81]-[83] and more recently in [65] and [84]-[89]. The bulk of the available inhomogeneous treatments are Newtonian, with general relativistic treatments only a recent addition to the literature. The evolution of linear density and vorticity perturbations in magnetic Newtonian cosmologies were addressed by [90]-93], and again more recently by [94,95]; general relativistic treatments have been given in [96]-98]. These investigations have established that magnetic fields are 
sources of density and vorticity distortions, but the rather complicated action of the $B$-field did not allow for analytic solutions to the relativistic equations. Solutions were provided with the help of covariant and gauge-invariant techniques, which considerably simplify the mathematics of cosmological magnetohydrodynamics [99]-[102]. The covariant equations were also used as the basis for qualitative studies by means of phase-plane methods [103], while an analogous approach has been used to analyse two-component charged plasmas [104 105. In addition to the linear regime, a number of authors have looked into the non-linear interaction between cosmic magnetic fields and electrically-charged fluids, primarily on sub-horizon scales. This includes work on the amplification of the $B$-field by shearing effects during the anisotropic collapse of a proto-galactic cloud [106]-[116]. The aforementioned studies combine analytical and numerical approaches, and apply to the pre- or to the post-recombination era of the universe. All this has motivated and facilitated work on the potential effects of magnetism on the CMB spectrum, including its polarisation [47,63]- [80], and the low-quadrupole moment problem [117].

In the present article, we use covariant and gauge-invariant techniques to analyse the effects of cosmological magnetic fields on the large-scale structure of the universe. We do so by refining and extending the work of [99]-[101. After a brief introduction to the $1+3$ covariant formalism in $\S 2$, we use $\S 3$, 4 and 5 to provide a detailed discussion of cosmological electrodynamics and magnetohydrodynamics in full general relativity. Section 6 utilises this information to study the effects of the $B$-field on weakly magnetic almost-Friedmann-Robertson-Walker (FRW) cosmologies in a gauge-invariant manner. One of the key results is that linear perturbations in the magnetic energy density evolve in step with those in the density of the matter. Focusing on scalar perturbations, we look at the evolution of linearized matter perturbations during the radiation and dust eras in $\S 7$. During the radiation era, and on super-horizon scales, our solutions show the magnetic pressure slows down the standard 'Jeans' growth rate of the density contrast. At the same time, the $B$-field increases the oscillation frequency of smallscale density perturbations in proportion to its strength relative to the radiation background density. The same amount of increase is also observed in the associated Jeans length. After the radiation era ends, the fluid pressure is effectively zero, and the magnetic field emerges as the sole source of pressure support. We calculate the associated 'magnetic' Jeans length and find that for $\mu G$ fields its scale is comparable to the size of a galaxy cluster. Given that during the dust era the magnetic relative strength decays with time, we also calculate the late-time magnetic effects on density perturbations. Here too, we find that the magnetic pressure inhibits the gravitational clumping of matter. This section closes by looking at the evolution of magnetic density perturbations within an inflationary false-vacuum environment. We find that the outcome is very sensitive to the effective equation of state of the cosmic medium. Section 8 looks at the magnetic effects on isocurvature density fluctuations, namely on perturbations evolving on irrotational spatial hypersurfaces of constant curvature. After establishing the necessary conditions for the existence of such disturbances in the presence of the $B$-field, we find that they always decay in time. In $\S 9$, we treat the magnetic medium as a two-fluid system and consider the effective entropy perturbations coming from differences in the dynamical behaviour of the two components. Our study shows that such disturbances vanish during the radiation era, while after recombination they either vanish, or remain constant, depending on the initial conditions. The magnetic effects on rotational (vector) density per- 
turbations are considered in $\S 10$. At the linear perturbative level these distortions are directly related to the amount of vorticity in the universe. Focusing on the dust era, we show that the magnetic presence reduces the standard decay rate of rotational perturbations. This leads to more residual vorticity relative to magnetic-free universes. The implications of the $B$-field for the evolution of gravitational waves are examined in $\S$ 11. As a first step, we introduce the additional constraints needed to isolate the linear pure-tensor modes in the presence of a magnetic field. Once these have been imposed, we consider the magnetic effects on the propagation of gravitational-wave distortions. We also look at the role of the magnetic anisotropic pressure during the radiation era and discuss the critical, zero eigenvalue, case that emerges there.

\section{$2 \quad 1+3$ covariant description}

The covariant approach to general relativity and cosmology dates back to the work of Heckmann, Schücking, and Raychaudhuri in the 1950s [118,119] and it has since been employed in numerous applications by many authors. The formalism uses the kinematic quantities of the fluid, its energy density and pressure and the gravito-electromagnetic tensors instead of the metric, which in itself does not provide a covariant description. The key equations are the Ricci and Bianchi identities, applied to the fluid 4-velocity vector, while Einstein's equations are incorporated via algebraic relations between the Ricci and the energy-momentum tensors. Here, we will only give a brief description of the approach and direct the reader to a number of review articles for further details and references [120]-[123].

\subsection{Local spacetime splitting}

Consider a general spacetime with a Lorentzian metric $g_{a b}$ of signature $(-,+,+,+)$. Introduce a family of fundamental observers along a timelike congruence of worldlines tangent to the 4 -velocity vector 1

$$
u^{a}=\frac{\mathrm{d} x^{a}}{\mathrm{~d} \tau},
$$

where $\tau$ is the associated proper time and $u_{a} u^{a}=-1$ [122]. This fundamental velocity field introduces a local, 1+3 'threading' of the spacetime into time and space. The vector $u_{a}$ determines the time direction, and the tensor $h_{a b}=g_{a b}+u_{a} u_{b}$ projects orthogonal to 4-velocity field into what is known as the observer's instantaneous rest space. In the absence of rotation, $u_{a}$ is hypersurface orthogonal and $h_{a b}$ acts as the metric of the 3-D spatial sections.

Employing $u_{a}$ and $h_{a b}$, we define the covariant time derivative and the orthogonally projected

$\overline{1}$ Latin indices run between 0 and 3 and Greek vary from 1 to 3 . We use geometrized units with $c=1=8 \pi G$, which means that all geometrical variables have physical dimensions that are integer powers of length. 
gradient of any given tensor field $S_{a b \cdots}{ }^{c d \cdots}$ according to

$$
\dot{S}_{a b \cdots}{ }^{c d \cdots}=u^{e} \nabla_{e} S_{a b \cdots}{ }^{c d \cdots} \quad \text { and } \quad \mathrm{D}_{e} S_{a b \cdots}{ }^{c d \cdots}=h_{e}{ }^{s} h_{a}{ }^{f} h_{b}{ }^{p} h_{q}{ }^{c} h_{r}{ }^{d} \cdots \nabla_{s} S_{f p \cdots}{ }^{q r \cdots}
$$

respectively. The former indicates differentiation along the timelike direction and the latter operates on the observer's rest space.

\subsection{Matter fields}

Relative to the fundamental observers, the energy-momentum tensor of a general imperfect fluid decomposes into its irreducible parts as [122]

$$
T_{a b}=\rho u_{a} u_{b}+p h_{a b}+2 q_{(a} u_{b)}+\pi_{a b} .
$$

Here, $\rho=T_{a b} u^{a} u^{b}$ and $p=T_{a b} h^{a b} / 3$ are, respectively, the energy density and the isotropic pressure of the medium, $q_{a}=-h_{a}{ }^{b} T_{b c} u^{c}$ is the energy-flux vector relative to $u_{a}$, and $\pi_{a b}=$ $h_{\langle a}{ }^{c} h_{b\rangle}{ }^{d} T_{c d}$ is the symmetric and trace-free tensor that describes the anisotropic pressure of the fluid 2 It follows that $q_{a} u^{a}=0=\pi_{a b} u^{a}$. When the fluid is perfect, both $q_{a}$ and $\pi_{a b}$ are identically zero, and the remaining degrees of freedom are determined by the equation of state. For a barotropic medium the latter reduces to $p=p(\rho)$, with $c_{s}^{2}=\mathrm{d} p / \mathrm{d} \rho$ giving the the square of the associated adiabatic sound speed.

When dealing with a multi-component medium, or when allowing for peculiar velocities, one needs to account for the velocity 'tilt' between the matter components and the fundamental observers (e.g. see [124]-[128]). Here we will consider a single-component fluid and we will assume that the fundamental observers move with it.

\subsection{Covariant kinematics}

The observers' motion is characterized by the irreducible kinematical quantities of the $u_{a^{-}}$ congruence, which emerge from the covariant decomposition of the 4-velocity gradient

$$
\nabla_{b} u_{a}=\sigma_{a b}+\omega_{a b}+\frac{1}{3} \Theta h_{a b}-A_{a} u_{b}
$$

where $\sigma_{a b}=\mathrm{D}_{\langle b} u_{a\rangle}, \omega_{a b}=\mathrm{D}_{[b} u_{a]}, \Theta=\nabla^{a} u_{a}=\mathrm{D}^{a} u_{a}$ and $A_{a}=u^{b} \nabla_{b} u_{a}$ are respectively the shear and the vorticity tensors, the volume expansion (or contraction) scalar, and the 4acceleration vector [122]. Then, $\sigma_{a b} u^{a}=0=\omega_{a b} u^{a}=A_{a} u^{a}$ by construction. The volume scalar is used to introduce a representative length scale (the cosmological scale factor $a$ ) by means of definition $\dot{a} / a=\Theta / 3$. Also, on using the orthogonally projected alternating tensor $\varepsilon_{a b c}$ (with

2 Angled brackets denote the symmetric and trace-free part of second-rank tensors projected orthogonally to $u_{a}$ and the projected component of vectors (i.e. $v_{\langle a\rangle}=h_{a}{ }^{b} v_{b}$ ). 
$\dot{\varepsilon}_{a b c}=3 u_{[a} \varepsilon_{b c] d} A^{d}$ - see [135]), one defines the vorticity vector $\omega_{a}=\varepsilon_{a b c} \omega^{b c} / 2$. Note that $\epsilon_{a b c}=$ $\eta_{a b c d} u^{d}$, where $\eta_{a b c d}$ is the totally antisymmetric permutation tensor of the spacetime. This is a covariantly constant quantity, with $\eta_{a b c d} \eta^{e f p q}=-4 ! \delta_{[a}{ }^{e} \delta_{b}{ }^{f} \delta_{c}{ }^{p} \delta_{d]}{ }^{q}$ and $\eta^{0123}=\left[-\operatorname{det}\left(g_{a b}\right)\right]^{-1 / 2}$. The tensor $v_{a b}=\mathrm{D}_{b} u_{a}=\sigma_{a b}+\omega_{a b}+(\Theta / 3) h_{a b}$ describes the relative motion of neighbouring observers (with the same 4 -velocity). In particular, $v_{a}=v_{a b} \chi^{b}$ is the relative velocity of the associated worldlines and $\chi_{a}$ is their relative position vector (see [121]129] for details).

The non-linear covariant kinematics is determined by a set of three propagation equations complemented by an equal number of constraints [122]. The former contains Raychaudhuri's formula

$$
\dot{\Theta}=-\frac{1}{3} \Theta^{2}-\frac{1}{2}(\rho+3 p)-2\left(\sigma^{2}-\omega^{2}\right)+\mathrm{D}^{a} A_{a}+A_{a} A^{a},
$$

for the time evolution of $\Theta$; the shear propagation equation

$$
\dot{\sigma}_{\langle a b\rangle}=-\frac{2}{3} \Theta \sigma_{a b}-\sigma_{c\langle a} \sigma_{b\rangle}^{c}-\omega_{\langle a} \omega_{b\rangle}+\mathrm{D}_{\langle a} A_{b\rangle}+A_{\langle a} A_{b\rangle}-E_{a b}+\frac{1}{2} \pi_{a b},
$$

which describes kinematical anisotropies; and the evolution equation of the vorticity

$$
\dot{\omega}_{\langle a\rangle}=-\frac{2}{3} \Theta \omega_{a}-\frac{1}{2} \operatorname{curl} A_{a}+\sigma_{a b} \omega^{b} .
$$

Here $\sigma^{2}=\sigma_{a b} \sigma^{a b} / 2$ and $\omega^{2}=\omega_{a b} \omega^{a b} / 2=\omega_{a} \omega^{a}$ are respectively the scalar magnitudes of the shear and the vorticity, while $E_{a b}$ is the electric component of the Weyl tensor (see $\S 4.2$ below). Also, curl $v_{a}=\varepsilon_{a b c} \mathrm{D}^{b} v^{c}$ for any orthogonally projected vector $v_{a}$.

Equations (2.3.2), (2.3.3) and (2.3.4) are complemented by a set of three non-linear constraints. These are the shear

the vorticity

$$
\mathrm{D}^{b} \sigma_{a b}=\frac{2}{3} \mathrm{D}_{a} \Theta+\operatorname{curl} \omega_{a}+2 \varepsilon_{a b c} A^{b} \omega^{c}-q_{a},
$$

$$
\mathrm{D}^{a} \omega_{a}=A_{a} \omega^{a}
$$

and the magnetic Weyl constraint

$$
H_{a b}=\operatorname{curl} \sigma_{a b}+\mathrm{D}_{\langle a} \omega_{b\rangle}+2 A_{\langle a} \omega_{b\rangle},
$$

where curl $T_{a b} \equiv \varepsilon_{c d\langle a} \mathrm{D}^{c} T_{b\rangle}{ }^{d}$ for any symmetric, orthogonally projected tensor $T_{a b}$. We finally note that the cosmological constant has been set to zero (i.e. $\Lambda=0$ ) throughout this review.

\section{Electromagnetic fields}

Covariant studies of electromagnetic fields date back to the work of Ehlers [120] and Ellis [129]. In addition to its inherent mathematical compactness and clarity, the formalism facilitates a physically intuitive fluid description of the Maxwell field. This is represented as an imperfect fluid with properties specified by its electric and magnetic components. For a fully covariant study of electromagnetic fields in curved spacetimes the reader is referred to [130]. 


\subsection{Electric and magnetic components}

The Maxwell field is covariantly characterized by the antisymmetric electromagnetic (Faraday) tensor $F_{a b}$. Relative to a fundamental observer, the latter decomposes as [129]

$$
F_{a b}=2 u_{[a} E_{b]}+\varepsilon_{a b c} B^{c},
$$

where $E_{a}=F_{a b} u^{b}$ and $B_{a}=\varepsilon_{a b c} F^{b c} / 2$ are respectively the electric and magnetic fields measured by the observer. Note that $E_{a} u^{a}=0=B_{a} u^{a}$, ensuring that both $E_{a}$ and $B_{a}$ are spacelike vectors. Also, $B_{a}=\varepsilon_{a b c} F^{b c} / 2$ guarantees that $B_{a}$ is the dual of $F_{a b}$.

The Faraday tensor also determines the energy-momentum tensor of the Maxwell field according to

$$
T_{a b}^{(e m)}=-F_{a c} F_{b}^{c}-\frac{1}{4} F_{c d} F^{c d} g_{a b} .
$$

The above combines with (3.1.1) to facilitate the irreducible decomposition of $T_{a b}^{(e m)}$ relative to the $u_{a}$-frame [129],

$$
T_{a b}^{(e m)}=\frac{1}{2}\left(E^{2}+B^{2}\right) u_{a} u_{b}+\frac{1}{6}\left(E^{2}+B^{2}\right) h_{a b}+2 \mathcal{Q}_{(a} u_{b)}+\mathcal{P}_{a b} .
$$

Here $E^{2}=E_{a} E^{a}$ and $B^{2}=B_{a} B^{a}$ are the magnitudes of the two fields, $\mathcal{Q}_{a}=\varepsilon_{a b c} E^{b} B^{c}$ is the electromagnetic Poynting vector and $\mathcal{P}_{a b}$ is a symmetric, trace-free tensor given by

$$
\mathcal{P}_{a b}=\mathcal{P}_{\langle a b\rangle}=\frac{1}{3}\left(E^{2}+B^{2}\right) h_{a b}-E_{a} E_{b}-B_{a} B_{b} .
$$

Expression (3.1.3) provides a fluid description of the Maxwell field and manifests its generically anisotropic nature. In particular, the electromagnetic field corresponds to an imperfect fluid with energy density $\left(E^{2}+B^{2}\right) / 2$, isotropic pressure $\left(E^{2}+B^{2}\right) / 6$, anisotropic stresses given by $\mathcal{P}_{a b}$ and an energy-flux vector represented by $\mathcal{Q}_{a}$. Equation (3.1.3) also ensures that $T_{a}^{(e m)} a=0$, in agreement with the trace-free nature of the radiation stress-energy tensor. Finally, we note that by putting the isotropic and anisotropic pressure together one arrives at the familiar Maxwell tensor, which assumes the covariant form

$$
\mathcal{M}_{a b}=\frac{1}{2}\left(E^{2}+B^{2}\right) h_{a b}-E_{a} E_{b}-B_{a} B_{b} .
$$

\subsection{Maxwell's equations}

We follow the evolution of the electromagnetic field by means of Maxwell's equations. In their standard tensor form these read

$$
\nabla_{[c} F_{a b]}=0 \quad \text { and } \quad \nabla^{b} F_{a b}=J_{a}
$$


where (3.2.1a) reflects the existence of a 4-potential and $J_{a}$ is the 4-current that sources the electromagnetic field. With respect to the $u_{a}$-congruence, the 4-current splits into its irreducible parts according to

$$
J_{a}=\rho_{e} u_{a}+\mathcal{J}_{a},
$$

with $\rho_{e}=-J_{a} u^{a}$ representing the measurable charge density and $\mathcal{J}_{a}=h_{a}{ }^{b} J_{b}$ the orthogonally projected current (i.e. $\mathcal{J}_{a} u^{a}=0$ ).

Relative to a fundamental observer, each one of Maxwell's equations decomposes into a timelike and a spacelike component. Projecting (3.2.1 a) and (3.2.1 b) along and orthogonal to the 4 -velocity vector $u_{a}$, one obtains a set of two propagation equations [129]

$$
\begin{aligned}
& \dot{E}_{\langle a\rangle}=\left(\sigma_{a b}+\varepsilon_{a b c} \omega^{c}-\frac{2}{3} \Theta h_{a b}\right) E^{b}+\varepsilon_{a b c} A^{b} B^{c}+\operatorname{curl} B_{a}-\mathcal{J}_{a}, \\
& \dot{B}_{\langle a\rangle}=\left(\sigma_{a b}+\varepsilon_{a b c} \omega^{c}-\frac{2}{3} \Theta h_{a b}\right) B^{b}-\varepsilon_{a b c} A^{b} E^{c}-\operatorname{curl} E_{a},
\end{aligned}
$$

and the following pair of constraints

$$
\begin{aligned}
& \mathrm{D}^{a} E_{a}=\rho_{e}-2 \omega^{a} B_{a}, \\
& \mathrm{D}^{a} B_{a}=2 \omega^{a} E_{a} .
\end{aligned}
$$

Note that, in addition to the usual 'curl' and 'divergence' terms, Eqs. (3.2.3)-(3.2.6) also contain effects triggered by the relative motion of neighbouring observers (with the same 4velocity - see $\S 2.3$ ). These are carried by the kinematic terms in the right-hand side of the above. Thus, $\tilde{\rho}_{e}=-2 \omega_{a} B^{a}$ is an effective electric charge caused by the relative motion of the magnetic field, while $2 \omega^{a} E_{a}$ acts as an effective magnetic charge triggered by the relatively moving $E$-field. The acceleration terms in (3.2.3) and (3.2.4), on the other hand, also reflect the fact that spacetime is treated as a single entity.

\subsection{Conservation laws}

The twice contracted Bianchi identities guarantee the conservation of the total energy momentum tensor, namely that $\nabla^{b} T_{a b}=0$. This constraint splits into a timelike and a spacelike part, which respectively lead to the energy density and the momentum-density conservation laws. The energy momentum tensor of the electromagnetic field satisfies the constraint $\nabla^{b} T_{a b}^{(e m)}=-F_{a b} J^{b}$, with the Faraday tensor given by (3.1.1) and the quantity in the righthand side representing the Lorentz 4 -force. Thus, for charged matter the conservation of the total energy-momentum tensor $T_{a b}=T_{a b}^{(m)}+T_{a m}^{(\mathrm{em})}$ leads to the formulae

$$
\dot{\rho}=-\Theta(\rho+p)-\mathrm{D}^{a} q_{a}-2 A^{a} q_{a}-\sigma^{a b} \pi_{a b}+E_{a} \mathcal{J}^{a},
$$

for the energy density, and 


$$
\begin{aligned}
(\rho+p) A_{a}= & -\mathrm{D}_{a} p-\dot{q}_{\langle a\rangle}-\frac{4}{3} \Theta q_{a}-\left(\sigma_{a b}+\omega_{a b}\right) q^{b}-\mathrm{D}^{b} \pi_{a b}-\pi_{a b} A^{b} \\
& +\rho_{e} E_{a}+\varepsilon_{a b c} \mathcal{J}^{b} B^{c}
\end{aligned}
$$

for the momentum density. The last two terms in the right-hand side of (3.3.2) represent the familiar form of the Lorentz force. We also note that the electromagnetic effects depend on the electrical properties of the medium (see $\S 3.4$ below).

The antisymmetry of the Faraday tensor (see Eq. (3.1.1)) and the second of Maxwell's formulae (see Eq. (3.2.1b)) imply $\nabla^{a} J_{a}=0$ and therefore ensure the conservation of the 4-current density. Using decomposition (3.2.2), we arrive at the covariant form of the charge-density conservation law 129

$$
\dot{\rho}_{e}=-\Theta \rho_{e}-\mathrm{D}^{a} \mathcal{J}_{a}-A^{a} \mathcal{J}_{a} .
$$

Hence, in the absence of spatial currents, the evolution of the charge density depends entirely on the volume expansion (or contraction) of the fluid element.

\subsection{Ohm's law}

The relation between the 4-current and the electric field, as measured by the fundamental observers, is determined by Ohm's law. Following [131,132], the latter has the covariant form

$$
J_{a}=\rho_{e} u_{a}+\varsigma E_{a}
$$

where $\varsigma$ is the scalar conductivity of the medium. Thus, Ohm's law splits the 4-current into a timelike convective component and a conducting spacelike counterpart. Projecting (3.4.1) into the observer's rest space gives

$$
\mathcal{J}_{a}=\varsigma E_{a}
$$

This form of Ohm's law covariantly describes the resistive magnetohydrodynamic (MHD) approximation in the single-fluid approach. Note the absence of the induced electric field from the above, reflecting the fact that the covariant form of Maxwell's formulae (see expressions (3.2.3)-(3.2.6) ) already incorporates the effects of relative motion. According to (3.4.2), nonzero spatial currents are compatible with a vanishing electric field as long as the conductivity of the medium is infinite (i.e. for $\varsigma \rightarrow \infty$ ). Thus, at the limit of ideal magnetohydrodynamics, the electric field vanishes in the frame of the fluid. On the other hand, zero electrical conductivity implies that the spatial currents vanish, even when the electric field is non-zero. The electrical conductivity is typically treated in a phenomenological manner and here we will also assume that it remains constant throughout the medium.

\section{Gravitational field}

Covariantly, the local gravitational field is described by a set of algebraic relations between the Ricci curvature tensor and the energy-momentum tensor of the matter. The free gravitational 
field, on the other hand, is described by the electric and magnetic components of the conformal curvature (Weyl) tensor.

\subsection{Local Ricci curvature}

In the general-relativistic geometrical interpretation of gravity, matter determines the spacetime curvature, which in turn dictates the motion of the matter. This interaction is evident in the Einstein field equations, which in the absence of a cosmological constant take the form

$$
R_{a b}=T_{a b}-\frac{1}{2} T g_{a b}
$$

where $R_{a b}=R_{a c b}^{c}$ is the spacetime Ricci tensor, $T_{a b}$ is the energy-momentum tensor of the matter fields, with $T=T_{a}^{a}$ being the trace. For our purposes the total energy-momentum

tensor has the form $T_{a b}=T_{a b}^{(f)}+T_{a b}^{(e m)}$, where $T_{a b}^{(f)}$ is given by Eq. (2.2.1) and $T_{a b}^{(e m)}$ by (3.1.3). Thus,

$$
\begin{aligned}
T_{a b}= & {\left[\rho+\frac{1}{2}\left(B^{2}+E^{2}\right)\right] u_{a} u_{b}+\left[p+\frac{1}{6}\left(B^{2}+E^{2}\right)\right] h_{a b}+2\left(q_{(a}+\mathcal{Q}_{(a}\right) u_{b)} } \\
& +\pi_{a b}+\mathcal{P}_{a b}
\end{aligned}
$$

ensuring that $\rho+\left(B^{2}+E^{2}\right) / 2$ is the total energy density of the system, $p+\left(B^{2}+E^{2}\right) / 6$ is the total isotropic pressure, $q_{a}+\mathcal{Q}_{a}$ is the total heat-flux vector and $\pi_{a b}+\mathcal{P}_{a b}$ is the total anisotropic pressure. The inclusion of electromagnetic terms in the energy-momentum tensor of the matter guarantees that the contribution of the Maxwell field to the spacetime geometry is fully accounted for.

The successive contraction of the Einstein field equations, assuming that $T_{a b}$ is given by Eq. (4.1.2), leads to the following algebraic relations:

$$
\begin{aligned}
R_{a b} u^{a} u^{b} & =\frac{1}{2}\left(\rho+3 p+E^{2}+B^{2}\right), \\
h_{a}{ }^{b} R_{b c} u^{c} & =-\left(q_{a}+\mathcal{Q}_{a}\right) \\
h_{a}{ }^{c} h_{b}{ }^{d} R_{c d} & =\left\{\frac{1}{2}\left[\rho-p+\frac{1}{3}\left(E^{2}+B^{2}\right)\right]\right\} h_{a b}+\pi_{a b}+\mathcal{P}_{a b} .
\end{aligned}
$$

In addition, the trace of (4.1.1) gives $R=-T$, with $R=R_{a}{ }^{a}$ and $T=T_{a}{ }^{a}=3 p-\rho$, where the latter result is guaranteed by the trace-free nature of $T_{a b}^{(\mathrm{em})}$. Note that the above expressions are valid irrespective of the strength of the electromagnetic components and recall that $q_{a}=0=\pi_{a b}$ when dealing with a perfect fluid. 


\subsection{Long-range Weyl curvature}

The Ricci tensor describes the local gravitational field of the nearby matter. The long-range gravitational field, namely gravitational waves and tidal forces, propagates through the Weyl conformal curvature tensor. The splitting of the gravitational field into its local and non-local components is demonstrated in the following decomposition of the Riemann tensor,

$$
R_{a b c d}=C_{a b c d}+\frac{1}{2}\left(g_{a c} R_{b d}+g_{b d} R_{a c}-g_{b c} R_{a d}-g_{a d} R_{b c}\right)-\frac{1}{6} R\left(g_{a c} g_{b d}-g_{a d} g_{b c}\right),
$$

where $C_{a b c d}$ is the Weyl tensor. This shares all the symmetries of the Riemann tensor and is also trace-free (i.e. $C^{c}{ }_{a c b}=0$ ). Relative to the fundamental observers, the Weyl tensor decomposes into its irreducible parts according to

$$
C_{a b c d}=\left(g_{a b q p} g_{c d s r}-\eta_{a b q p} \eta_{c d s r}\right) u^{q} u^{s} E^{p r}-\left(\eta_{a b q p} g_{c d s r}+g_{a b q p} \eta_{c d s r}\right) u^{q} u^{s} H^{p r},
$$

where $\eta_{a b c d}=\eta_{[a b c d]}$ is the spacetime permutation tensor defined in $\S 2.3$ and $g_{a b c d}=g_{a c} g_{b d}-$ $g_{a d} g_{b c}$ (e.g. see [133[134]). The symmetric and trace-free tensors $E_{a b}$ and $H_{a b}$ are known as the electric and magnetic Weyl components and they are given by

$$
E_{a b}=C_{a c b d} u^{c} u^{d} \quad \text { and } \quad H_{a b}=\frac{1}{2} \varepsilon_{a}{ }^{c d} C_{c d b e} u^{e},
$$

with $E_{a b} u^{b}=0=H_{a b} u^{b}$. Given that $E_{a b}$ has a Newtonian counterpart, the electric part of the Weyl tensor is associated with the tidal gravitational field. The magnetic component, on the other hand, has no Newtonian analogue and is therefore primarily associated with gravitational waves and spatial 3-curvature anisotropy [122]. Of course, both tensors are required if gravitational waves are to exist.

The Weyl tensor represents the part of the curvature that is not determined locally by matter. However, the dynamics of the Weyl field are not entirely arbitrary because the Riemann tensor satisfies the Bianchi identities. When contracted, the latter take the form [133]

$$
\nabla^{d} C_{a b c d}=\nabla_{[b} R_{a] c}+\frac{1}{6} g_{c[b} \nabla_{a]} R,
$$

by means of decomposition (4.2.1). In one sense the contracted Bianchi identities act as the field equations for the Weyl tensor, determining the part of the spacetime curvature that depends on the matter distribution at other points [133]. The form of the contracted Bianchi identities guarantees that once the electromagnetic contribution to the Ricci curvature has been incorporated, through the Einstein field equations, the effect of the Maxwell field on the Weyl curvature has also been fully accounted for.

The $1+3$ splitting of (4.2.4) provides a set of two propagation and two constraint equations for the evolution of the long-range gravitational field, namely of tidal forces and gravity waves. In particular, on using the decomposition (4.2.2), the timelike component of (4.2.4) leads to [122]135] 


$$
\begin{aligned}
\dot{E}_{\langle a b\rangle}= & -\Theta E_{a b}-\frac{1}{2}\left[\rho+p+\frac{2}{3}\left(B^{2}+E^{2}\right)\right] \sigma_{a b}+\operatorname{curl} H_{a b}-\frac{1}{2}\left(\dot{\pi}_{a b}+\dot{\mathcal{P}}_{a b}\right) \\
& -\frac{1}{6} \Theta\left(\pi_{a b}+\mathcal{P}_{a b}\right)-\frac{1}{2} \mathrm{D}_{\langle a}\left(q_{b\rangle}+\mathcal{Q}_{b\rangle}\right)-A_{\langle a}\left(q_{b\rangle}+\mathcal{Q}_{b\rangle}\right) \\
& +3 \sigma_{\langle a}{ }^{c}\left[E_{b\rangle c}-\frac{1}{6}\left(\pi_{b\rangle c}+\mathcal{P}_{b\rangle c}\right)\right]+2 \varepsilon_{c d\langle a} A^{c} H_{b\rangle}{ }^{d} \\
& -\varepsilon_{c d\langle a} \omega^{c}\left[E_{b\rangle}{ }^{d}+\frac{1}{2}\left(\pi_{b\rangle}{ }^{d}+\mathcal{P}_{b\rangle}{ }^{d}\right)\right]
\end{aligned}
$$

and

$$
\begin{aligned}
\dot{H}_{\langle a b\rangle}= & -\Theta H_{a b}-\operatorname{curl} E_{a b}+\frac{1}{2}\left(\operatorname{curl} \pi_{a b}+\operatorname{curl} \mathcal{P}_{a b}\right)+3 \sigma_{\langle a}{ }^{c} H_{b\rangle c}-\frac{3}{2} \omega_{\langle a}\left(q_{b\rangle}+\mathcal{Q}_{b\rangle}\right) \\
& -2 \varepsilon_{c d\langle a} A^{c} E_{b\rangle}{ }^{d}+\varepsilon_{c d\langle a}\left[\frac{1}{2} \sigma_{b\rangle}^{c}\left(q^{d}+\mathcal{Q}^{d}\right)-\omega^{c} H_{b\rangle}{ }^{d}\right] .
\end{aligned}
$$

Taking the time derivatives of the above one arrives to a pair of wavelike equations for the electric and the magnetic parts of the Weyl tensor, showing how curvature distortions propagate in the form of gravitational waves like ripples in the spacetime fabric. These waves are also subjected to a set of constraints, which emerge from the spacelike component of the decomposed Eq. (4.2.4) and are given by [122,135]

$$
\begin{aligned}
\mathrm{D}^{b} E_{a b}= & \frac{1}{3} \mathrm{D}_{a}\left[\rho+\frac{1}{2}\left(B^{2}+E^{2}\right)\right]-\frac{1}{2} \mathrm{D}^{b}\left(\pi_{a b}+\mathcal{P}_{a b}\right)-\frac{1}{3} \Theta\left(q_{a}+\mathcal{Q}_{a}\right)+\frac{1}{2} \sigma_{a b}\left(q^{b}+\mathcal{Q}^{b}\right) \\
& -3 H_{a b} \omega^{b}+\varepsilon_{a b c}\left[\sigma^{b}{ }_{d} H^{c d}-\frac{3}{2} \omega^{b}\left(q^{c}+\mathcal{Q}^{c}\right)\right]
\end{aligned}
$$

and

$$
\begin{aligned}
\mathrm{D}^{b} H_{a b}= & {\left[\rho+p+\frac{2}{3}\left(B^{2}+E^{2}\right)\right] \omega_{a}-\frac{1}{2} \operatorname{curl}\left(q_{a}+\mathcal{Q}_{a}\right)+3 E_{a b} \omega^{b}-\frac{1}{2}\left(\pi_{a b}+\mathcal{P}_{a b}\right) \omega^{b} } \\
& -\varepsilon_{a b c} \sigma_{d}^{b}\left[E^{c d}+\frac{1}{2}\left(\pi^{c d}+\mathcal{P}^{c d}\right)\right]
\end{aligned}
$$

respectively. The above expressions are similar to Maxwell's formulae, which explains the names of $E_{a b}$ and $H_{a b}$. In fact, this Maxwell-like form of the free gravitational field underlines the rich correspondence between electromagnetism and general relativity, which has been the subject of theoretical debate for many decades (see [136]-[139] for a representative list).

\subsection{Spatial curvature}

When the fluid is irrotational, the rest spaces of the fundamental observers mesh together to form spacelike surfaces orthogonal to their worldlines. These are normal to the $u_{a}$-congruence

and define the hypersurfaces of simultaneity of all the comoving observers. In the presence 
of vorticity, however, Frobenius' theorem forbids the existence of such integrable surfaces (e.g. see [140,141]). The $u_{a}$-congruence is no longer hypersurface orthogonal. Then, one can still talk about the observers' spatial rest-space but only locally. The local 3-Riemann tensor, defined by

$$
\mathcal{R}_{a b c d}=h_{a}{ }^{q} h_{b}{ }^{s} h_{c}{ }^{f} h_{d}^{p} R_{q s f p}-v_{a c} v_{b d}+v_{a d} v_{b c}
$$

where $v_{a b}=\mathrm{D}_{b} u_{a}$ is the relative position tensor. On using Eqs. (4.1.3)-(4.1.5) and expressions (4.2.1), (4.2.2), definition (4.3.1) gives

$$
\begin{aligned}
\mathcal{R}_{a b c d}= & -\varepsilon_{a b q} \varepsilon_{c d s} E^{q s}+\frac{1}{3}\left[\rho+\frac{1}{2}\left(E^{2}+B^{2}\right)-\frac{1}{3} \Theta^{2}\right]\left(h_{a c} h_{b d}-h_{a d} h_{b c}\right) \\
& +\frac{1}{2}\left[h_{a c}\left(\pi_{b d}+\mathcal{P}_{b d}\right)+\left(\pi_{a c}+\mathcal{P}_{a c}\right) h_{b d}-h_{a d}\left(\pi_{b c}+\mathcal{P}_{b c}\right)-\left(\pi_{a d}+\mathcal{P}_{a d}\right) h_{b c}\right] \\
& -\frac{1}{3} \Theta\left[h_{a c}\left(\sigma_{b d}+\omega_{b d}\right)+\left(\sigma_{a c}+\omega_{a c}\right) h_{b d}-h_{a d}\left(\sigma_{b c}+\omega_{b c}\right)-\left(\sigma_{a d}+\omega_{a d}\right) h_{b c}\right] \\
& -\left(\sigma_{a c}+\omega_{a c}\right)\left(\sigma_{b d}+\omega_{b d}\right)+\left(\sigma_{a d}+\omega_{a d}\right)\left(\sigma_{b c}+\omega_{b c}\right)
\end{aligned}
$$

which offers an irreducible decomposition of the local 3-Riemann tensor orthogonal to $u_{a}$. Then, one can easily show that

and that

$$
\mathcal{R}_{a b c d}=\mathcal{R}_{[a b][c d]}
$$

$$
\begin{aligned}
\mathcal{R}_{a b c d}-\mathcal{R}_{c d a b}= & -\frac{2}{3} \Theta\left(h_{a c} \omega_{b d}+\omega_{a c} h_{b d}-h_{a d} \omega_{b c}-\omega_{a d} h_{b c}\right) \\
& -2\left(\sigma_{a c} \omega_{b d}+\omega_{a c} \sigma_{b d}-\sigma_{a d} \omega_{b c}-\omega_{a d} \sigma_{b c}\right) .
\end{aligned}
$$

Therefore, in the absence of vorticity, $\mathcal{R}_{a b c d}=\mathcal{R}_{c d a b}$ and the spatial Riemann tensor possesses all the symmetries of its 4-D counterpart.

In analogy with $R_{a b}$ and $R$, the local Ricci tensor and Ricci scalar of the 3-D space orthogonal to $u_{a}$ are defined by

$$
\mathcal{R}_{a b}=h^{c d} \mathcal{R}_{c a d b}=\mathcal{R}^{c}{ }_{a c b} \quad \text { and } \quad \mathcal{R}=h^{a b} \mathcal{R}_{a b},
$$

respectively. Thus, contracting (4.3.2) along the first and third indices we arrive at what is usually referred to as the Gauss-Codacci equation:

$$
\begin{aligned}
\mathcal{R}_{a b}= & E_{a b}+\frac{2}{3}\left[\rho+\frac{1}{2}\left(E^{2}+B^{2}\right)-\frac{1}{3} \Theta^{2}+\sigma^{2}-\omega^{2}\right] h_{a b}+\frac{1}{2}\left(\pi_{a b}+\mathcal{P}_{a b}\right) \\
& -\frac{1}{3} \Theta\left(\sigma_{a b}+\omega_{a b}\right)+\sigma_{c\langle a} \sigma_{b\rangle}^{c}-\omega_{c\langle a} \omega_{b\rangle}^{c}+2 \sigma_{c[a} \omega_{b]}^{c} .
\end{aligned}
$$

A further contraction leads to the trace of the above and provides the local Ricci scalar of the 3-dimension space orthogonal to $u_{a}$

$$
\mathcal{R}=h^{a b} \mathcal{R}_{a b}=2\left[\rho+\frac{1}{2}\left(E^{2}+B^{2}\right)-\frac{1}{3} \Theta^{2}+\sigma^{2}-\omega^{2}\right] .
$$


This expression is of major importance, since it is nothing else but the generalized Friedmann equation. Indeed, when the electromagnetic terms and those measuring anisotropy are removed, the above assumes its familiar FRW form. Note that, on using (4.3.7), the GaussCodacci formula (see Eq. (4.3.6) ) reads

$$
\mathcal{R}_{a b}=\frac{1}{3} \mathcal{R} h_{a b}+E_{a b}+\frac{1}{2}\left(\pi_{a b}+\mathcal{P}_{a b}\right)-\frac{1}{3} \Theta\left(\sigma_{a b}+\omega_{a b}\right)+\sigma_{c\langle a} \sigma_{b\rangle}^{c}-\omega_{c\langle a} \omega_{b\rangle}^{c}+2 \sigma_{c[a} \omega_{b]}^{c}
$$

The latter may be used to calculate the curvature of the 3-space along a chosen direction. For instance, $\mathcal{R}_{a b} B^{a} B^{b}$ gives the 3-curvature distortions along the magnetic field lines.

\section{Non-linear cosmological magnetohydrodynamics}

With the exception of any period of inflation and early reheating, the universe has been a good conductor throughout its lifetime. As a result, $B$-fields of cosmological origin must have remained frozen into the expanding cosmic fluid during most of their evolution. This allows us to study the magnetic effects on structure formation within the ideal-MHD limits.

\subsection{Ideal MHD approximation}

Consider a general spacetime filled with a single barotropic fluid of very high conductivity. Ohm's law (see Eq. (3.4.2) ) guarantees that in the frame of the fundamental observer the electric field vanishes despite the presence of non-zero currents. Therefore, in the ideal MHD limit the energy-momentum tensor of the residual magnetic field simplifies to [99]

$$
T_{a b}^{(B)}=\frac{1}{2} B^{2} u_{a} u_{b}+\frac{1}{6} B^{2} h_{a b}+\Pi_{a b}
$$

with

$$
\Pi_{a b}=\Pi_{\langle a b\rangle}=\frac{1}{3} B^{2} h_{a b}-B_{a} B_{b} .
$$

Accordingly, the $B$-field corresponds to an imperfect fluid with energy density $\rho_{B}=B^{2} / 2$, isotropic pressure $p_{B}=B^{2} / 6$, and anisotropic stresses represented by the symmetric and trace-free tensor $\Pi_{a b}$.

Similarly, in the absence of an electric field, Maxwell's equations reduce to a single propagation formula, namely the covariant magnetic induction equation,

$$
\dot{B}_{\langle a\rangle}=\left(\sigma_{a b}+\varepsilon_{a b c} \omega^{c}-\frac{2}{3} \Theta h_{a b}\right) B^{b}
$$

and the following three constraints 


$$
\begin{aligned}
\operatorname{curl} B_{a} & =\mathcal{J}_{a}-\varepsilon_{a b c} A^{b} B^{c}, \\
\omega^{a} B_{a} & =\frac{1}{2} \rho_{e} \\
\mathrm{D}^{a} B_{a} & =0
\end{aligned}
$$

The right-hand side of (5.1.3) is due to the relative motion of the neighbouring observes and guarantees that the magnetic field lines always connect the same matter particles [99. This means that the field remains frozen-in with the highly conducting fluid. Expression (5.1.4) provides a direct relation between the spatial currents, which are responsible for keeping the field lines frozen-in with the matter, and the magnetic field itself (e.g. see [14]). Note Eq. (5.1.5) which shows that rotating neighbouring observers will measure a non-zero charge density, triggered by their relative motion, unless $\omega^{a} B_{a}=0$. Finally, (5.1.6) demonstrates that in the absence of magnetic monopoles the field lines remain closed and $B_{a}$ is a solenoidal vector.

\subsection{Magnetic evolution}

The magnetic induction equation also provides the non-linear evolution law for the energy density of the field. More precisely, contracting Eq. (5.1.3) with $B_{a}$ and then using (5.1.2) we arrive at

$$
\left(B^{2}\right)^{\cdot}=-\frac{4}{3} \Theta B^{2}-2 \sigma_{a b} \Pi^{a b}
$$

This shows that in a highly conducting cosmic medium we have $B^{2} \propto a^{-4}$ always unless there is substantial anisotropy, in which case the $B$-field behaves as an anisotropic radiative fluid. In fact, in a homogeneous and anisotropic radiation-dominated universe this latter situation arises even when the anisotropy is small because close to isotropy $\Pi^{a b} \propto \rho$ and the evolution of $B^{2}$ is determined at second-order with $B^{2} / \rho_{\text {rad }} \rightarrow \sigma / \Theta \propto 1 / \log (t)$ during the radiation era (see 6165] and also 66, 102]).

The nonlinear evolution of the anisotropic magnetic stresses comes from the time derivative of (5.1.2), which by means of Eqs. (5.1.3) and (5.2.1) leads to

$$
\dot{\Pi}_{a b}=-\frac{4}{3} \Theta \Pi_{a b}-\frac{2}{3} B^{2} \sigma_{a b}+2 \sigma_{c\langle a} \Pi_{b\rangle}^{c}-2 \omega_{c\langle a} \Pi_{b\rangle}^{c}
$$

\subsection{Conservation laws}

The energy momentum tensor corresponding to a magnetic single perfect fluid of infinite conductivity is given by

$$
T_{a b}=\left(\rho+\frac{1}{2} B^{2}\right) u_{a} u_{b}+\left(p+\frac{1}{6} B^{2}\right) h_{a b}+\Pi_{a b},
$$


Accordingly, the medium corresponds to an imperfect fluid with effective density equal to $\rho+B^{2} / 2$, isotropic pressure given by $p+B^{2} / 6$, zero heat flux and solely magnetic anisotropic stresses represented by $\Pi_{a b}($ see $(5.1 .2))$.

When applied to the above, and using the MHD form of Maxwell's equations, the standard conservation law $\nabla^{b} T_{a b}=0$ decomposes into the following expressions that respectively describe the energy-density

$$
\dot{\rho}=-(\rho+p) \Theta
$$

and the momentum-density

$$
\left(\rho+p+\frac{2}{3} B^{2}\right) A_{a}=-\mathrm{D}_{a} p-\varepsilon_{a b c} B^{b} \operatorname{curl} B^{c}-\Pi_{a b} A^{b}
$$

conservation [99,101]. Note the absence of magnetic terms in Eq. (5.3.2). This is guaranteed by the magnetic induction equation (5.1.3) and reflects the fact that the magnetic energy density is separately conserved $\sqrt[3]{3}$ Also, when there are no pressure gradients, (5.3.3) gives $A_{a} B^{a}=0$ to ensure that the field exerts no forces along its own direction. Finally, the left-hand side of (5.3.3) shows that the magnetic contribution to the total inertial mass of the system is $2 B^{2} / 3$.

Contracting Eq. (5.3.3) along $B_{a}$ we find that the contribution of the $B$-field to the momentum density vanishes, thus guaranteeing that the magnetic Lorentz force is always normal to the field lines. Also, the second term in the right-hand side of (5.3.3) decomposes as

$$
\varepsilon_{a b c} B^{b} \operatorname{curl} B^{c}=\frac{1}{2} \mathrm{D}_{a} B^{2}-B^{b} \mathrm{D}_{b} B_{a} .
$$

The last term in the above is the result of the magnetic tension. In so far as this tension stress is not balanced by the pressure gradients, the field lines are out of equilibrium and there is a non-zero Lorentz force acting on the particles of the magnetic fluid.

\subsection{Magnetic tension}

The anisotropic nature of magnetic fields is encoded in the energy-momentum tensor of the field - and particularly in the anisotropic pressure tensor (see (5.1.2)). This ensures that the $B$-fields exert a positive pressure orthogonal to their own direction, while carrying a tension along $B_{a}$. Both of these very well known magnetic features are reflected in the eigenvalues of $\Pi_{a b}$, which are positive $(1 / 3)$ perpendicular to $B_{a}$ and negative $(-2 / 3)$ parallel to it.

The magnetic tension also demonstrates the elasticity of the field lines and their tendency to remain as straight as possible by reacting to any effect that distorts them from equilibrium [13,16]. Within the context of general relativity, we can see this tendency by looking

3 Expressions (5.3.2) and (5.3.3) can also be obtained from the conservation laws (3.3.1) and (3.3.2). This is done by taking the ideal-MHD limit of the latter, using Eq. (5.1.4) and assuming perfect-fluid matter. 
at the effect of the field on the spatial curvature of a magnetic spacetime. In particular, contracting (4.3.6) along $B_{a}$ twice, and ignoring all sources but the magnetic field, we find that [142]

$$
\mathcal{R}_{a b} B^{a} B^{b}=\frac{1}{3} B^{4}+\frac{1}{2} \Pi_{a b} B^{a} B^{b}=0,
$$

given that $\Pi_{a b} B^{b}=-\left(2 B^{2} / 3\right) B_{a}$ (see (5.1.2) $)$. Accordingly, despite the magnetic presence and the energy density contribution of the field, the curvature of the 3-space in the direction of the magnetic force lines is zero. Mathematically speaking, it is the contribution of the negative magnetic pressure along $B_{a}$ which cancels out the positive input of the field's energy density. More intuitively, however, one could argue that it is the elasticity of the magnetic lines, and their tendency to remain straight, that maintains the zero curvature along $B_{a}$.

In the presence of sources, $\mathcal{R}_{a b} B^{a} B^{b}$ is generally non-zero and the magnetic lines are forced out of equilibrium. When allowing for spatial inhomogeneities, the reaction of the field's tension, as expressed through the second stress in the right-hand side of (5.3.4), to these geometrically induced distortions generally leads to counter-intuitive effects. The potential implications of these tension stresses, for cosmology as well as astrophysics, have been discussed in [143][146]. In spatially homogeneous spacetimes on the other hand, like the Bianchi $I$ models, the magnetic Lorentz force vanishes and the field's tension is manifested only as a negative energy-density input, through the anisotropic pressure tensor $\left(\Pi_{a b}-\right.$ see Eq. (5.1.2) $)$. Such cosmologies were recently investigated in [89].

\subsection{Kinematical evolution}

The magnetic presence affects the kinematics of the highly conducting medium both directly and indirectly. In particular, Raychaudhuri's equation reads

$$
\dot{\Theta}=-\frac{1}{3} \Theta^{2}-\frac{1}{2}\left(\rho+3 p+B^{2}\right)-2\left(\sigma^{2}-\omega^{2}\right)+\mathrm{D}^{a} A_{a}+A_{a} A^{a},
$$

showing that the field's contribution to the total gravitational mass is $B^{2}$. There are also indirect magnetic effects propagating through the rest of the terms in the right-hand side of (5.5.1). Of these effects, probably the most important are carried by the acceleration terms which follow from Eq. (5.3.3). Similarly, there are direct and indirect magnetic effects on the shear evolution. In the magnetic environment the propagation equation of the shear tensor takes the form

$$
\dot{\sigma}_{\langle a b\rangle}=-\frac{2}{3} \Theta \sigma_{a b}-\sigma_{c\langle a} \sigma_{b\rangle}^{c}-\omega_{\langle a} \omega_{b\rangle}+\mathrm{D}_{\langle a} A_{b\rangle}+A_{\langle a} A_{b\rangle}-E_{a b}+\frac{1}{2} \Pi_{a b},
$$

which reveals how the anisotropic pressure of the field directly induces and also affects kinematical anisotropies. Rotation, on the other hand hand, is affected only indirectly through the magnetic effects on the 4-acceleration of the fluid. To be precise,

$$
\dot{\omega}_{\langle a\rangle}=-\frac{2}{3} \Theta \omega_{a}-\frac{1}{2} \operatorname{curl} A_{a}+\sigma_{a b} \omega^{b} .
$$


This means that the $B$-field not only affects the rotational behaviour of the observers' frame but it can also generate vorticity.

The kinematical constraints are also only indirectly affected by the field's presence. This is manifested by the absence of explicit magnetic terms in Eqs. (2.3.5)-(2.3.7). When applied to a highly conducting barotropic fluid expressions (2.3.6) and (2.3.7) remain unchanged, while (2.3.5) reduces to

$$
\mathrm{D}^{b} \sigma_{a b}=\frac{2}{3} \mathrm{D}_{a} \Theta+\operatorname{curl} \omega_{a}+2 \varepsilon_{a b c} A^{b} \omega^{c} .
$$

When $\mathrm{D}^{a} \sigma_{a b}=0$, the shear is divergence-free and its pure-tensor part has been isolated. In other words, there is no way of using $\sigma_{a b}$ to construct a scalar or a vector. This condition will be imposed later, in $\S 11$, when studying the magnetic effects of gravitational-wave perturbations.

\subsection{Spacetime curvature}

The energy-momentum tensor of a highly conducting magnetic perfect fluid is given by Eqs. (5.1.1), (5.1.2). In that case formulae (4.1.3)-(4.1.5) reduce to

$$
R_{a b} u^{a} u^{b}=\frac{1}{2}\left(\rho+3 p+B^{2}\right), \quad \quad h_{a}{ }^{b} R_{b c} u^{c}=0
$$

and

$$
h_{a}{ }^{c} h_{b}{ }^{d} R_{c d}=\left[\frac{1}{2}\left(\rho-p+\frac{1}{3} B^{2}\right)\right] h_{a b}+\Pi_{a b},
$$

respectively. We note that the above are fully non-linear expressions and that the former quantifies the total gravitational mass of the MHD fluid (in agreement with (5.5.1)).

We evaluate the magnetic contribution to the local 3-Ricci tensor orthogonal to the $u_{a^{-}}$ congruence, by taking the MHD limit of the geometrical relations given in $\S 4.3$. Expressions (4.3.6) and (4.3.7), in particular, simplify to

$$
\begin{aligned}
\mathcal{R}_{a b}= & E_{a b}+\frac{2}{3}\left(\rho+\frac{1}{2} B^{2}-\frac{1}{3} \Theta^{2}+\sigma^{2}-\omega^{2}\right) h_{a b}+\frac{1}{2} \Pi_{a b}-\frac{1}{3} \Theta\left(\sigma_{a b}+\omega_{a b}\right) \\
& +\sigma_{c\langle a} \sigma_{b\rangle}^{c}-\omega_{c\langle a} \omega^{c}{ }_{b\rangle}+2 \sigma_{c[a} \omega_{b]}^{c}
\end{aligned}
$$

and

$$
\mathcal{R}=2\left(\rho+\frac{1}{2} B^{2}-\frac{1}{3} \Theta^{2}+\sigma^{2}-\omega^{2}\right),
$$

respectively. This is simply the familiar Friedmann equation generalized to the magnetic and highly conducting environment. 


\subsection{Evolution of inhomogeneities}

\subsubsection{Basic variables}

Spatial inhomogeneities of a given physical quantity are covariantly described by its orthogonally projected gradients. For our purposes, the basic variables are the comoving fractional gradients,

$$
\Delta_{a}=\frac{a}{\rho} \mathrm{D}_{a} \rho \quad \text { and } \quad \mathcal{B}_{a}=\frac{a}{B^{2}} \mathrm{D}_{a} B^{2},
$$

describing spatial variations in the fluid and the magnetic energy density distributions (see [147][149]) and 99,100 respectively). Both variables are dimensionless by construction, and vanish identically when the spacetime is spatially homogeneous. As we will show later, $\Delta_{a}$ and $\mathcal{B}_{a}$ respectively describe spatial variations in the fluid and the magnetic energy densities, as measured by a pair of neighbouring fundamental observers, in a gauge-invariant way (see $\S$ 6.3.1).

The above are supplemented by a pair of auxiliary variables, which describe spatial inhomogeneities in the average expansion and the isotropic pressure of the fluid. These are [147]

$$
\mathcal{Z}_{a}=a \mathrm{D}_{a} \Theta \quad \text { and } \quad Y_{a}=\mathrm{D}_{a} p,
$$

respectively. It should be noted that, for barotropic fluids, the variable $Y_{a}$ is directly related to $\Delta_{a}$ and therefore is redundant for all practical purposes. Finally, one may also monitor spatial inhomogeneities in the distribution of the magnetic field vector by means of the orthogonally projected tensor 99]

$$
\mathcal{M}_{a b}=\mathrm{D}_{b} B_{a} .
$$

This is trace-free in the ideal MHD limit (see constraint (5.1.6)) but, contrary to the gradients (5.7.1) and (5.7.2), it does not vanish unless the FRW metric is spatially flat (see Appendix A in [100] and also $\S 6.3 .2$ here).

\subsubsection{Evolution equations}

In the presence of magnetic fields, the non-linear evolution of spatial inhomogeneities in the density distribution of a single, highly conducting perfect fluid is described by the expression

$$
\begin{aligned}
\dot{\Delta}_{\langle a\rangle}= & w \Theta \Delta_{a}-(1+w) \mathcal{Z}_{a}+\frac{a \Theta}{\rho} \varepsilon_{a b c} B^{b} \operatorname{curl} B^{c}+\frac{2}{3} c_{\mathrm{a}}^{2}(1+w) a \Theta A_{a} \\
& -\left(\sigma_{b a}+\omega_{b a}\right) \Delta^{b}+\frac{a \Theta}{\rho} \Pi_{a b} A^{b},
\end{aligned}
$$

with $w=p / \rho$ and $c_{\mathrm{a}}^{2}=B^{2} / \rho(1+w)$ representing the Alfvén speed (see $\S 6.1 .4$ below). This equation has been obtained by taking the proper-time derivative of $(5.7 .1 \mathrm{a})$ and then projecting it onto the observer's 3-dimensional instantaneous rest space. In the process, we have also employed the conservation laws (5.3.2) and (5.3.3). 
Similarly, starting from definition (5.7.2 a) and using (5.3.3), (5.5.1), we arrive at the following non-linear evolution equation for the expansion gradients

$$
\begin{aligned}
\dot{\mathcal{Z}}_{\langle a\rangle}= & -\frac{2}{3} \Theta \mathcal{Z}_{a}-\frac{1}{2} \rho \Delta_{a}-\frac{1}{2} B^{2} \mathcal{B}_{a}+\frac{3}{2} a \varepsilon_{a b c} B^{b} \operatorname{curl} B^{c}+a \mathrm{D}_{a} A \\
& +\left[\frac{1}{2} \mathcal{R}-3\left(\sigma^{2}-\omega^{2}\right)+A+A_{b} A^{b}\right] a A_{a}+2 a A^{b} \mathrm{D}_{a} A_{b} \\
& -\left(\sigma_{b a}+\omega_{b a}\right) \mathcal{Z}^{b}+\frac{3}{2} a \Pi_{a b} A^{b}-2 a \mathrm{D}_{a}\left(\sigma^{2}-\omega^{2}\right)
\end{aligned}
$$

where $A=\mathrm{D}^{a} A_{a}$ and $\mathcal{R}$ is the Ricci scalar of the observer's local 3-D rest space.

Finally, the orthogonally-projected time derivative of (5.7.1 b) leads to the non-linear propagation formula monitoring spatial inhomogeneities in the magnetic energy density

$$
\begin{aligned}
\dot{\mathcal{B}}_{\langle a\rangle}= & \frac{4}{3(1+w)} \dot{\Delta}_{\langle a\rangle}-\frac{4 w \Theta}{3(1+w)} \Delta_{a}-\frac{4 a \Theta}{3 \rho(1+w)} \varepsilon_{a b c} B^{b} \operatorname{curl} B^{c}-\frac{4}{3} a \Theta\left(1+\frac{2}{3} c_{\mathrm{a}}^{2}\right) A_{a} \\
& -\left(\sigma_{b a}+\omega_{b a}\right) \mathcal{B}^{b}+\frac{4}{3(1+w)}\left(\sigma_{b a}+\omega_{b a}\right) \Delta^{b}-\frac{4 a \Theta}{3 \rho(1+w)} \Pi_{a b} A^{b}-\frac{2 a}{B^{2}} \Pi^{b c} \mathrm{D}_{a} \sigma_{b c} \\
& -\frac{2 a}{B^{2}} \sigma^{b c} \mathrm{D}_{a} \Pi_{b c}+\frac{2}{B^{2}} \sigma_{b c} \Pi^{b c} \mathcal{B}_{a}-\frac{2 a}{B^{2}} \sigma_{b c} \Pi^{b c} A_{a} .
\end{aligned}
$$

In deriving the above, we have employed the non-linear relations (5.1.3) and (5.2.1), as well as (5.7.4). This helped to eliminate the expansion gradients from the right-hand sideof Eq. (5.7.6). We emphasize that (5.7.6) cannot beused when the equation of state of the cosmic medium has the vacuumenergy form contributed by a cosmological constant in the Einsteinequations, namely for $w=p / \rho=-1$. In that case we can no longeruse (5.7.4) to eliminate $\mathcal{Z}_{a}$ from (5.7.6) and the evolution of density inhomogeneities is described by a different system of equations (see $\oint 7.5)$.

\section{Perturbed magnetic FRW cosmologies}

The symmetries of FRW spacetimes cannot naturally accommodate generic anisotropic sources like magnetic fields. This means that when based on a Friedmann background, any magnetic study will require some degree of approximation. Nevertheless, it is intuitively plausible that sufficiently weak $B$-fields can be adequately studied within perturbed almost-FRW models. This belief, which has been at the core of almost every study of cosmological magnetic fields, has been confirmed by the analysis of exact [65] and perturbed magnetic Bianchi $I$ universes [102]. 


\subsection{Background evolution}

\subsubsection{Zero-order approach}

We consider a spatially flat Friedmann background and allow for the presence of a weak magnetic field. This is effectively a test field that does not disrupt isotropy and has $B^{2} \ll \rho$. The background $B$-field can be either homogeneous or sufficiently random, so that $\left\langle B_{a}\right\rangle=$ $0 \neq\left\langle B^{2}\right\rangle$ (with $\left\langle B^{2}\right\rangle$ homogeneous on all the scales of interest). In both cases the magnetic anisotropic stress $\Pi_{a b}$ is treated as a first-order perturbation and the only zero-order magnetic variable is $B^{2}$. First-order perturbations arise through $\Pi_{a b}$, the spatial gradients $\mathrm{D}_{b} B^{2}$ and the spatial derivatives $\mathrm{D}_{b} B_{a}$. If the background test field is homogeneous, the scalar $B^{2}$ describes its local density and pressure. For a stochastic test field, $B^{2}$ describes the average density and pressure of the background field. In either case, the form of the linearized MHD equations guarantees that the magnetic effects propagate only via $B^{2}$-terms (see $\S 7.2$ ) and that, at least when studying scalar (i.e. density) perturbations, the two approaches lead to the same linear equations and results.

Alternatively, one can treat the magnetic field as a first-order perturbation, usually as a stochastic Gaussian field (e.g. see [76,80]). One can adapt our analysis to this scheme by removing the magneto-curvature terms from all the linear formulae (see $\S$ [7.2).

\subsubsection{Zero-order equations}

Locally, the evolution of the background is governed by the standard Friedmann and Raychaudhuri equations. Introducing $H=\dot{a} / a$ as our zero-order Hubble parameter, these are

$$
3 H^{2}=\rho \quad \text { and } \quad \dot{H}=-H^{2}-\frac{1}{6} \rho(1+3 w),
$$

and are supplemented by the conservation laws for the matter and the magnetic energy densities, namely by

$$
\dot{\rho}=-3 H \rho(1+w) \quad \text { and } \quad\left(B^{2}\right)^{\cdot}=-4 H B^{2},
$$

respectively 4 This is obtained from the magnetic induction equation (see (5.1.3) ), which guarantees that

$$
\dot{B}_{a}=-2 H B_{a}
$$

Note that the weakness of the $B$-field allows us to neglect the magnetic contribution to the total energy density and gravitational mass of the system. This explains the absence of magnetic terms from the right-hand sides of (6.1.1 a) and (6.1.1b), provided $w \neq-1 / 3$ in the latter case.

4 The spatially averaged magnetic energy density depends only on time and evolves in tune with its local counterpart. Indeed, using the local result $(6.1 .2 \mathrm{~b})$, it is straightforward to show that $\left\langle B^{2}\right\rangle^{\circ}=$ $-4 H\left\langle B^{2}\right\rangle$ on any background domain because $\langle H\rangle=H$ in FRW models (e.g. see [150] for details). 
Also, $(6.1 .2 \mathrm{~b})$ guarantees the radiation-like evolution $B^{2} \propto a^{-4}$ for the zero-order field, which means that the background magnetic flux is conserved.

\subsubsection{Specifying the magnetic medium}

The nature of the cosmic medium is determined by its equation of state. Here, we will be dealing with a highly conducting barotropic fluid with $p=w \rho$, where $w$ is the barotropic index. This vanishes in the case of dust, takes the value $w=1 / 3$ for radiation and indicates a stiff fluid when $w=1$. Also, for $w<-1 / 3$ the total gravitational mass of the matter is negative and the model enters a period of accelerated expansion, which corresponds to exponential de Sitter-type inflation when $w=-1$. We find that

$$
\dot{w}=-(1+w)\left(c_{s}^{2}-w\right) \Theta
$$

where $c_{s}^{2}=\mathrm{d} p / \mathrm{d} \rho$ is the square of the adiabatic sound speed. Therefore, a time-invariant barotropic index in a non-static universe (i.e. $\dot{w}=0$ and $\Theta \neq 0$ ) means that $c_{s}^{2}=w=$ constant, and vice versa.

\subsubsection{Magnetic strength and Alfvén speed}

The magnetic strength, relative to the matter component, is measured by the dimensionless energy-density ratio $\beta=B^{2} / \rho$. Then, given the weakness of the field (i.e. since $\beta \ll 1$ ), the square of the Alfvén speed is conveniently defined as

$$
c_{\mathrm{a}}^{2}=\frac{\beta}{1+w}
$$

ensuring that $c_{\mathrm{a}}^{2} \ll 1$ at all times. The only exception is when $w \rightarrow-1$, in which case the proper definition for the Alfvén speed is $c_{\mathrm{a}}^{2}=\beta /(1+w+\beta) 5$ Thus, from now on, $w \neq-1$ unless stated otherwise. On using (6.1.4) and Eqs. (6.1.2), the time derivative of the above gives

$$
\left(c_{\mathrm{a}}^{2}\right)^{\cdot}=-\left(1-3 c_{s}^{2}\right) H c_{\mathrm{a}}^{2},
$$

to zero order. Accordingly, the Alfvén speed does not change with time when $c_{s}^{2}=1 / 3$, which agrees with the radiation-like linear evolution of the magnetic energy density in almost-FRW environments, 6

$\overline{5}$ In the standard literature the square of the Alfvén speed is $c_{\mathrm{a}}^{2}=B^{2} / \rho=\beta$ (e.g. see [132]).

6 When shear anisotropy is included, the parameter $\beta$ and therefore $c_{\mathrm{a}}^{2}$ are no longer constant during the radiation-dominated epoch but display a slow, 'quasi static', logarithmic decay (see [61,65] and also [66 102]). 


\subsection{Linear evolution}

We linearize the full equations by treating quantities with non-zero background values as zero-order variables in perturbative terms. On the other hand, quantities that vanish in the background are of order one and higher-order terms are ignored. Therefore, the only zero-order quantities in our case are the fluid energy density $(\rho)$ and pressure $(p)$, the magnetic energy density $\left(B^{2}\right)$, and the Hubble parameter $(H)$.

The linear conservation law of the magnetic energy density maintains its background form, given by Eq. (6.1.2b). Similarly, the linear propagation of the magnetic vector is still given by (6.1.3), which implies that $B_{a} \propto a^{-2}$ and that the magnetic flux remains conserved. On the other hand, the scalar $B^{a} \operatorname{curl} B_{a}$, which is used to measure the helicity of the field (e.g. see [71]), obeys the linear evolution law

$$
\left(B^{a} \operatorname{curl} B_{a}\right)^{\cdot}=-5 H B^{a} \operatorname{curl} B_{a} .
$$

The above means that the magnetic helicity decays as $a^{-5}$ and therefore it decreases faster than the energy density of the $B$-field (recall that $B^{2} \propto a^{-4}$ throughout the linear regime). Note that in deriving (6.2.1) we have assumed overall charge neutrality and used Eq. (6.1.3), together with the first-order expression $B^{a}\left(\operatorname{curl} B_{a}\right)^{\cdot}=-3 H B^{a} \operatorname{curl} B_{a}$, obtained by means of commutator (A.2) in $\S$ A.2.

To first order, the conservation law of the fluid energy-density retains its the background form (see Eq. (6.1.2 a)). However, when linearized around a FRW background, the momentumdensity conservation equation (5.3.3) reduces to

$$
(1+w)\left(1+\frac{2}{3} c_{\mathrm{a}}^{2}\right) \rho A_{a}=-c_{s}^{2} \mathrm{D}_{a} \rho-\varepsilon_{a b c} B^{b} \operatorname{curl} B^{c},
$$

with $\mathrm{D}_{a} p=c_{s}^{2} \mathrm{D}_{a} \rho$ due to the barotropic nature of the medium.

The magnetic field's presence affects the linear kinematics in a number of ways. The average

volume expansion, for example, is determined by the following version of the Raychaudhuri equation

$$
\dot{\Theta}=-\frac{1}{3} \Theta^{2}-\frac{1}{2} \rho(1+3 w+\beta)-\frac{c_{s}^{2}}{a(1+w)}\left(1-\frac{2}{3} c_{\mathrm{a}}^{2}\right) \mathrm{D}^{a} \Delta_{a}-\frac{c_{\mathrm{a}}^{2}}{2 a} \mathrm{D}^{a} \mathcal{B}_{a}+\frac{1}{3} c_{\mathrm{a}}^{2} \mathcal{R},
$$

where $\mathcal{R}=h^{a b} \mathcal{R}_{a b}$ is the 3 -Ricci scalar and we have kept up to $c_{\mathrm{a}}^{2}$-order terms. As expected, any local increase in the magnetic energy density will decelerate the linear expansion of the model in the same way that matter perturbations do. However, the $B$-field has an additional effect due to the magneto-curvature term in the right-hand side of (6.2.3). This further decelerates the expansion if $\mathcal{R}<0$, but tends to accelerate it when $\mathcal{R}$ is positive. This counter-intuitive behaviour, whichresults from the tension of the field, can have nontrivial and veryunexpected implications for spatially curved magnetic spacetimes [143]-[146]. 
The rotational behaviour of the weakly magnetic fluid is described by the vorticity propagation equation, which to linear order takes the form

$$
\dot{\omega}_{a}=-2\left[1-\frac{3}{2} c_{s}^{2}\left(1-\frac{2}{3} c_{\mathrm{a}}^{2}\right)-\frac{1}{6} c_{\mathrm{a}}^{2}\right] H \omega_{a}-\frac{1}{2 \rho(1+w)}\left(1-\frac{2}{3} c_{\mathrm{a}}^{2}\right) B^{b} \mathrm{D}_{b} \operatorname{curl} B_{a} .
$$

This means that the magnetic presence will generate vorticity unless the field's distribution is curl-free, or more specifically, unless curl $B_{a}$ remains unchanged along the direction of the magnetic force lines. Thus, $B$-fields can act as sources of rotation. The magnetic effect on pre-existing vorticesis more difficult to quantify. As we will see later, in section $\S$ 10.3, the presence of the field can slow down the standard decay rate of vorticity and therefore increase the residualamount of rotation relative to magnetic-free cosmologies. Recallthat in the absence of the $B$-field, vorticity always decays $\operatorname{unless}_{s}^{2}>2 / 3[151]$.

The evolution of linear kinematical anisotropies in the magnetic fluid is governed by the following expression of the shear propagation equation

$$
\begin{aligned}
\dot{\sigma}_{a b}= & -2 H \sigma_{a b}+\frac{1}{2} \Pi_{a b}-E_{a b}-\frac{c_{s}^{2}}{a(1+w)}\left(1-\frac{2}{3} c_{\mathrm{a}}^{2}\right) \mathrm{D}_{\langle a} \Delta_{b\rangle}-\frac{c_{\mathrm{a}}^{2}}{2 a} \mathrm{D}_{\langle a} \mathcal{B}_{b\rangle} \\
& +\frac{1}{\rho(1+w)}\left(1-\frac{2}{3} c_{\mathrm{a}}^{2}\right) B^{c} \mathrm{D}_{c} \mathrm{D}_{\langle a} B_{b\rangle}+\frac{1}{3} c_{\mathrm{a}}^{2} \mathcal{R}_{\langle a b\rangle} .
\end{aligned}
$$

The magnetic effects are diverse. The anisotropic pressure of the field is supplemented by anisotropies in the distribution of the magnetic vector and by those in the energy density of the $B$-field. In addition, there is a purely geometrical magneto-curvature effect, provided that $\mathcal{R}_{\langle a b\rangle}$ is non-zero at the linear level.

In the ideal MHD limit the kinematical variables also satisfy constraints (5.5.4), (2.3.6) and (2.3.7), which then linearize to

$$
\mathrm{D}^{b} \sigma_{a b}=\frac{2}{3} \mathrm{D}_{a} \Theta+\operatorname{curl} \omega_{a}, \quad \mathrm{D}^{a} \omega_{a}=0
$$

and

$$
H_{a b}=\operatorname{curl} \sigma_{a b}+\mathrm{D}_{\langle a} \omega_{b\rangle}
$$

respectively. According to the above, $\omega_{a}$ is a linear solenoidal vector and in the absence of rotation the magnetic Weyl component is fully determined by the shear tensor. Additional (geometrical) constraints between the kinematical and the dynamical quantities in the highly conducting medium are provided by (5.6.3) and (5.6.4). The respective linearized counterparts of these expressions are

$$
\mathcal{R}_{a b}=E_{a b}+\frac{2}{3}\left(\rho+\frac{1}{2} B^{2}-\frac{1}{3} \Theta^{2}\right) h_{a b}+\frac{1}{2} \Pi_{a b}-H\left(\sigma_{a b}+\omega_{a b}\right)
$$

and

$$
\mathcal{R}=2\left(\rho+\frac{1}{2} B^{2}-\frac{1}{3} \Theta^{2}\right)
$$




\subsection{Spatial inhomogeneities}

\subsubsection{Local interpretation of the inhomogeneity variables}

To linear order, the inhomogeneity variables defined in section 5.7 .1 describe measurable differences in the spatial distribution of physical quantities. In order to verify this, consider the relative position vector $\chi_{a}$ connecting the same two points on the worldlines of neighbouring fundamental observers. Following [122,147], we have $\chi_{a} u^{a}=0$ and

$$
\dot{\chi}_{a}=H \chi_{a}+\left(\sigma_{a b}+\omega_{a b}\right) \chi^{b}
$$

which means that in a FRW universe $\chi_{a}=a \chi_{a}^{0}$ (with $\chi_{a}^{0}=$ constant and $a_{0}=1$ ). If $P$ and $\tilde{P}$ are points on the two worldlines with coordinates $\left(x_{a}\right)$ and $\left(x_{a}+\chi_{a}\right)$ respectively and $\rho, \tilde{\rho}$ are the associated values of the energy density, a Taylor expansion around $P$ gives

$$
\tilde{\rho}-\rho=\chi^{a} \mathrm{D}_{a} \rho
$$

to leading order. Since $\chi_{a} \propto a$ in the FRW background and using definition (5.7.1 $\left.\mathrm{a}\right)$, the above translates into

$$
\delta \rho=\chi_{0}^{a} \Delta_{a},
$$

where $\delta \rho=(\tilde{\rho}-\rho) / \rho$. In other words, the comoving fractional gradient $\Delta_{a}$ describes the measurable local density variation between two neighbouring fundamental observers. Moreover, $\Delta_{a}$ closely corresponds to the familiar energy-density contrast of the non-covariant studies. The same analysis also applies to the rest of the variables defined in 5.7.1.

\subsubsection{Gauge invariance of the inhomogeneity variables}

Cosmological perturbations have long been known to suffer from gauge-related ambiguities, reflecting the fact that in perturbation theory one deals with two spacetime manifolds. The first is the physical spacetime $(\mathcal{W})$ that corresponds to the real universe, while the second $(\overline{\mathcal{W}})$ is a fictitious background described by an idealised mathematical model. To proceed we need to establish an one-to-one correspondence, namely a gauge $\phi: \overline{\mathcal{W}} \rightarrow \mathcal{W}$, between these two manifolds. Such point identification maps are generally arbitrary and the gauge problem stems from our inherent freedom to make gauge transformations. The latter differ from ordinary coordinate transformations because they also change the point identification between $\overline{\mathcal{W}}$ and $\mathcal{W}$.

We define perturbations as the difference between the value of a given quantity at some event in the realistic universe and its value at the corresponding (through the gauge) event in the background spacetime. Gauge transformations, however, generally change this correspondence and therefore the value of the perturbation itself. This makes perturbations gauge-dependent and arbitrary. For instance, we can select the gauge in such a way that a given perturbation vanishes [147]. Following the Stewart \& Walker lema, the simplest quantities that remain 
invariant under gauge-transformations are scalars that are constant in the background spacetime and tensors that vanish there [152, 7 Note that the same general criteria also apply to second-order perturbations, but this time the Stewart \& Walker requirements must be satisfied by the first-order variables [153].

In a Friedmann universe all physical variables are functions of cosmic time only, though they acquire additional spatial dependence in perturbed 'nearly-FRW' universes. As a result of the spatial homogeneity of the unperturbed Friedmann background, the variables (5.7.1) and (5.7.2) vanish to zero order. Consider the density gradient for example. Then,

$$
\mathrm{D}_{a} \rho=h_{a}^{b} \nabla_{b} \rho=h_{a}{ }^{0} \nabla_{0} \rho+h_{a}{ }^{\alpha} \nabla_{\alpha} \rho=0
$$

because $\nabla_{\alpha} \rho=0$ and in a comoving frame $h_{a}{ }^{0}=0$ (with $\alpha=1,2,3$ - see also [147]). This result guarantees the vanishing of the inhomogeneity variables (5.7.1), (5.7.2), in the background, and therefore ensures their gauge invariance at the linear perturbative level [152. Note, however, that the gauge invariance of $\mathrm{D}_{b} B_{a}$, and therefore of $\mathcal{M}_{a b}$, are guaranteed only in spatially flat FRW backgrounds [100]. One can see that by applying the 3-Ricci identity to a zero-order 3-vector $v_{a}$ (see Eq. (A.2) in $\S$ A.1). In the absence of rotation the latter reads

$$
2 \mathrm{D}_{[a} \mathrm{D}_{b]} v_{c}=\mathcal{R}_{a b c d} v^{d}
$$

which means that $\mathrm{D}_{b} v_{a} \neq 0$ unless $\mathcal{R}_{a b c d}=0[100]$.

\subsubsection{Irreducible inhomogeneity variables}

The orthogonally projected density gradient, $\Delta_{a}$, contains collective information about three types of inhomogeneities, namely density perturbations, vortices and shape-distortions. This information is encoded in the dimensionless comoving gradient

$$
\Delta_{a b}=a \mathrm{D}_{b} \Delta_{a}
$$

and can be decoded by splitting the latter into its irreducible components as follows [149,154]

$$
\Delta_{a b}=\frac{1}{3} a \mathrm{D}^{c} \Delta_{c} h_{a b}+a \mathrm{D}_{[b} \Delta_{a]}+a \mathrm{D}_{\langle b} \Delta_{a\rangle} .
$$

The quantities on the right-hand side are associated with the aforementioned three different types of inhomogeneity. The scalar $\mathrm{D}^{a} \Delta_{a}$ describes spatial variations in the matter density (i.e. overdensities or underdensities), the skew part is related to magnitude-preserving changes of $\Delta_{a}$ (i.e. rotations), and the symmetric and trace-free tensor $\mathrm{D}_{\langle b} \Delta_{a\rangle}$ describes shape distortions in the anisotropy pattern of the gradient field (e.g. pancakes or cigar-like structures).

$\overline{7}$ The only alternative way of constructing gauge-independent variables is by using tensors which are linear combinations of products of the Kronecker deldas with constant coefficients [152]. 
In an exactly analogous way, all the information regarding perturbations in the volume expansion and in the magnetic energy density is stored in the dimensionless second-rank tensors

$$
\mathcal{Z}_{a b}=a \mathrm{D}_{b} \mathcal{Z}_{a} \quad \text { and } \quad \mathcal{B}_{a b}=a \mathrm{D}_{b} \mathcal{B}_{a}
$$

respectively. These decompose into their irreducible parts according to [149,101]

$$
\mathcal{Z}_{a b}=\frac{1}{3} a \mathrm{D}^{c} \mathcal{Z}_{c} h_{a b}+a \mathrm{D}_{[b} \mathcal{Z}_{a]}+a \mathrm{D}_{\langle b} \mathcal{Z}_{a\rangle}
$$

and

$$
\mathcal{B}_{a b}=\frac{1}{3} a \mathrm{D}^{c} \mathcal{B}_{c} h_{a b}+a \mathrm{D}_{[b} \mathcal{B}_{a]}+a \mathrm{D}_{\langle b} \mathcal{B}_{a\rangle}
$$

\subsubsection{Linear equations}

When linearized around a FRW background permeated by a weak magnetic field, the nonlinear propagation equations of section 5.7 .2 reduce to

$$
\begin{aligned}
& \dot{\Delta}_{a}=3 w H \Delta_{a}-(1+w) \mathcal{Z}_{a}+\frac{3 a H}{\rho} \varepsilon_{a b c} B^{b} \operatorname{curl} B^{c}+2 c_{\mathrm{a}}^{2}(1+w) a H A_{a} \\
& \dot{\mathcal{Z}}_{a}=-2 H \mathcal{Z}_{a}-\frac{1}{2} \rho \Delta_{a}-\frac{1}{2} B^{2} \mathcal{B}_{a}+\frac{3}{2} a \varepsilon_{a b c} B^{b} \operatorname{curl} B^{c}+a \mathrm{D}_{a} A
\end{aligned}
$$

and

$$
\dot{\mathcal{B}}_{a}=\frac{4}{3(1+w)} \dot{\Delta}_{a}-\frac{4 w H}{1+w} \Delta_{a}-\frac{4 a H}{\rho(1+w)} \varepsilon_{a b c} B^{b} \operatorname{curl} B^{c}-4 a H\left(1+\frac{2}{3} c_{\mathrm{a}}^{2}\right) A_{a}
$$

respectively. Therefore, the linear evolution of the inhomogeneities depends on the 4-acceleration of the highly conducting matter. To first order, the latter satisfies the momentum-density conservation law (see Eq. (6.2.2))

$$
(1+w)\left(1+\frac{2}{3} c_{\mathrm{a}}^{2}\right) a \rho A_{a}=-c_{s}^{2} \rho \Delta_{a}-a \varepsilon_{a b c} B^{b} \operatorname{curl} B^{c}
$$

Using the commutation law $\mathrm{D}_{[a} \mathrm{D}_{b]} v_{c}=-\omega_{a b} \dot{v}_{\langle c\rangle}+\mathcal{R}_{d c b a} v^{d} / 2$ between the spatial gradients of $v_{a}$ (with $v_{a} u^{a}=0$ - see Eq. (A.2) in $\S$ A.1) ), and the constraint (5.1.6), the projected divergence of the above leads to

$$
A=-\frac{c_{s}^{2}}{a(1+w)}\left(1-\frac{2}{3} c_{\mathrm{a}}^{2}\right) \mathrm{D}^{a} \Delta_{a}-\frac{c_{\mathrm{a}}^{2}}{2 a} \mathrm{D}^{a} \mathcal{B}_{a}+\frac{1}{3} c_{\mathrm{a}}^{2} \mathcal{R}
$$

where

$$
\mathcal{R}=2\left(\rho+\frac{1}{2} B^{2}-\frac{1}{3} \Theta^{2}\right)
$$


is the linearized 3-Ricci scalar (see Eq. (6.2.9)). Substituting (6.3.14) into the right-hand side of (6.3.13) we arrive at

$$
\dot{\mathcal{B}}_{a}=\frac{4}{3(1+w)} \dot{\Delta}_{a}+\frac{4\left(c_{s}^{2}-w\right) H}{1+w} \Delta_{a}
$$

which means that linear inhomogeneities in the magnetic energy density are only affected by those of the matter. To close the system of (6.3.11), (6.3.12) and (6.3.17) we require the propagation equation of the 3-Ricci scalar. Taking the time derivative of (6.2.9) and linearising we obtain

$$
\dot{\mathcal{R}}=-2\left(1+\frac{2}{3} c_{\mathrm{a}}^{2}\right) H \mathcal{R}+\frac{4 c_{s}^{2}}{a(1+w)}\left(1-\frac{2}{3} c_{\mathrm{a}}^{2}\right) H \mathrm{D}^{a} \Delta_{a}+\frac{2 c_{\mathrm{a}}^{2} H}{a} \mathrm{D}^{a} \mathcal{B}_{a} .
$$

We note that for $c_{s}^{2}=w$, as it happens during the radiation and the dust epochs when the equation of state of the cosmic medium remains unchanged (i.e. when $\dot{w}=0$ - see Eq. (6.1.4)), the above expression reduces to

$$
\dot{\mathcal{B}}_{a}=\frac{4}{3(1+w)} \dot{\Delta}_{a}
$$

This guarantees that linear perturbations in the magnetic energy density evolve in step with those in the density of the highly conducting matter. When dealing with a radiative fluid the above gives $\dot{\mathcal{B}}_{a}=\dot{\Delta}_{a}$, while $\dot{\mathcal{B}}_{a}=4 \dot{\Delta}_{a} / 3$ for non-relativistic dust 8 The implication of (6.3.19) is that the effective entropy perturbations coming from the different dynamical behaviour of the two components (i.e. the fluid and the magnetic field) are constant, or zero (see section 9).

\section{Density perturbations}

\subsection{Basic variables}

Decomposition (6.3.7) allows for a fully covariant and gauge-invariant study of linear density perturbations, vortices and shape distortions. Here, we will focus on the scalar component of $\Delta_{a b}$. This is defined by

$$
\Delta=a \mathrm{D}^{a} \Delta_{a}
$$

and when positive (negative) it describes overdensities (underdensities) in the distribution of the perturbed fluid. In other words, $\Delta$ corresponds to the relative increase or decrease in the matter density as measured by a pair of neighbouring fundamental observers. Similarly, $\mathcal{Z}=a \mathrm{D}^{a} \mathcal{Z}_{a}$ and $\mathcal{B}=a \mathrm{D}^{a} \mathcal{B}_{a}$ describe scalar perturbations in the volume expansion and the magnetic energy density respectively.

$\overline{8}$ The covariantly derived result (6.3.19) is in complete agreement with relation $B \propto \rho^{2 / 3(1+w)}$, familiar from conventional studies of weakly magnetized FRW models. Indeed, taking the square of the above, and then its variation, we find that $\delta B^{2} / B^{2}=4 \delta \rho / 3 \rho(1+w)$ with $\delta B^{2} / B^{2}$ and $\delta \rho / \rho$ corresponding to $\mathcal{B}_{a}$ and $\Delta_{a}$ respectively. 
To linear order definition (7.1.1) reduces to $\Delta=\left(a^{2} / \rho\right) \mathrm{D}^{2} \rho$, while analogous expressions hold for $\mathcal{B}$ and $\mathcal{Z}$. In addition, it helps to introduce the following rescaling

$$
\mathcal{K}=a^{2} \mathcal{R}
$$

of the perturbed 3-Ricci scalar $\mathcal{R}$. This is also gauge invariant, given the spatial flatness of the FRW background.

\subsection{Linear equations}

Taking the orthogonally projected divergence of Eq. (6.3.11), using (6.3.14) and keeping up to $c_{\mathrm{a}}^{2}$-order terms, given the weakness of the magnetic field, we arrive at the following equation

$$
\dot{\Delta}=3 w\left(1-\frac{2}{3} c_{\mathrm{a}}^{2}\right) H \Delta-(1+w) \mathcal{Z}+\frac{3}{2} c_{\mathrm{a}}^{2}(1+w) H \mathcal{B}-c_{\mathrm{a}}^{2}(1+w) H \mathcal{K},
$$

for the linear evolution of $\Delta$. Note that in deriving the above we have also set $\dot{w}=0$ to zero order, which means that $w=c_{s}^{2}=$ constant in the background, and employed the linear relation $a \mathrm{D}^{a} \dot{\Delta}_{a}=\dot{\Delta}$. Following (7.2.1), the field will generally act as a source for density perturbations even when there are no such distortions present initially. Also, the magnetic field's presence has a direct and an indirect effect on $\Delta$. The former results from the pressure part of the Lorentz force (see decomposition (5.3.4) in $\S 5.3$ ) and carries the effects of the isotropic magnetic pressure. The latter comes from the tension component of the Lorentz force and it is triggered by the magnetic coupling to the spatial curvature of the perturbed model. Surprisingly, a positive 3 -curvature perturbation causes $\Delta$ to decrease, while a negative $\mathcal{K}$ has the opposite effect. This rather counter-intuitive behaviour of the magneto-curvature term in (7.2.1) is a direct consequence of the elasticity of the field lines (see also Eq. (7.2.2) below).

Similarly, using (6.3.15), together with the linear results $a \mathrm{D}^{a} \dot{\mathcal{Z}}_{a}=\dot{\mathcal{Z}}$ and $a \mathrm{D}^{a} \dot{\mathcal{B}}_{a}=\dot{\mathcal{B}}$, the linearized orthogonally-projected divergences of (6.3.12) and (6.3.17) lead to

$$
\begin{aligned}
\dot{\mathcal{Z}}= & -2\left(1+\frac{2}{3} c_{\mathrm{a}}^{2}\right) H \mathcal{Z}-\frac{1}{2} \rho\left(1-\frac{4}{3} c_{\mathrm{a}}^{2}\right) \Delta+\frac{1}{4} c_{\mathrm{a}}^{2}(1+w) \rho \mathcal{B}-\frac{1}{2} c_{\mathrm{a}}^{2}(1+w) \rho \mathcal{K} \\
& -\frac{c_{s}^{2}}{1+w}\left(1-\frac{2}{3} c_{\mathrm{a}}^{2}\right) \mathrm{D}^{2} \Delta-\frac{1}{2} c_{\mathrm{a}}^{2} \mathrm{D}^{2} \mathcal{B}
\end{aligned}
$$

and

$$
\dot{\mathcal{B}}=\frac{4}{3(1+w)} \dot{\Delta}+\frac{4\left(c_{s}^{2}-w\right) H}{1+w} \Delta,
$$

respectively. Finally, starting from the linear propagation equation of the 3-Ricci scalar we obtain

$$
\dot{\mathcal{K}}=-\frac{4}{3} c_{\mathrm{a}}^{2} H \mathcal{K}+\frac{4 c_{s}^{2}}{1+w}\left(1-\frac{2}{3} c_{\mathrm{a}}^{2}\right) H \Delta+2 c_{\mathrm{a}}^{2} H \mathcal{B}
$$


The system (7.2.1)-(7.2.4) describes the linear evolution of scalar inhomogeneities in the density distribution of the matter in a weakly magnetic and spatially flat almost-FRW universe 9 When the cosmic medium has a time-independent equation of state (i.e. for $\dot{w}=0$ ) we have $c_{s}^{2}=w=$ constant and Eq. (7.2.3) reduces to

$$
\dot{\mathcal{B}}=\frac{4}{3(1+w)} \dot{\Delta}
$$

It should be noted that in [99]-[101] factors of the form $1 \pm c_{\mathrm{a}}^{2}$ in the coefficients of the perturbed variables were set to unity, because of the overall weakness of the $B$-field. Here, retaining the factors $1 \pm c_{\mathrm{a}}^{2}$ in the system of (7.2.1)-(7.2.4) has improved the accuracy of our linear equations and will help us refine the results of [99]-[101]. When $1 \pm c_{\mathrm{a}}^{2} \simeq 1$, expressions (7.2.1)-(7.2.4) immediately reduce to their corresponding formulae of [99]-[101]. The reduction is obvious and straightforward for Eqs. (7.2.1)-(7.2.3) but not for (77.2.4). There, one needs to use definition (7.1.2) to show that $\dot{\mathcal{K}}+\left(4 c_{\mathrm{a}}^{2} / 3\right) H \mathcal{K} \simeq \dot{\mathcal{K}}$ when $1 \pm c_{\mathrm{a}}^{2} \simeq 1$. This requirement was overlooked in [103, resulting in the inconsistent linearisation of their equations.

\subsection{Evolution in the radiation era}

During the radiation epoch the background dynamics is determined by the parameters $w=$ $1 / 3=c_{s}^{2}, H=1 / 2 t$ and $\rho=3 / 4 t^{2}$. In addition, given the weakness of the magnetic field we have $c_{\mathrm{a}}^{2}=3 \beta / 4$, where $\beta=B^{2} / \rho=$ constant $\ll 1$. To proceed further we harmonically decompose the perturbed variables. In particular, we set $\Delta=\sum_{k} \Delta^{(k)} \mathcal{Q}_{(k)}$, where $\mathcal{Q}_{(k)}$ are the standard scalar harmonics (with $k$ representing the associated wavenumber) and $\mathrm{D}_{a} \Delta^{(k)}=0$. The harmonics are time-independent functions (i.e. $\dot{\mathcal{Q}}_{(k)}=0$ ) and satisfy the scalar LaplaceBeltrami equation

$$
\mathrm{D}^{2} \mathcal{Q}_{(k)}=-\left(\frac{k}{a}\right)^{2} \mathcal{Q}_{(k)} .
$$

Similarly, the rest of the perturbed variables decompose as $\mathcal{Z}=\mathcal{Z}^{(k)} \mathcal{Q}_{(k)}, \mathcal{B}=\mathcal{B}^{(k)} \mathcal{Q}_{(k)}$ and $\mathcal{K}=\mathcal{K}^{(k)} \mathcal{Q}_{(k)}$, with $\mathrm{D}_{a} \mathcal{Z}^{(k)}=0=\mathrm{D}_{a} \mathcal{B}^{(k)}=\mathrm{D}_{a} \mathcal{K}^{(k)}$. Implementing this harmonic decomposition, Eqs. (7.2.1)-(7.2.4) now read

$\overline{9}$ When $B^{2}$ is treated as a first order perturbation, the 3 -Ricci terms in the right-hand side of Eqs. (17.2.1), (7.2.2) are second order and therefore vanish at the linear level. In that case linearized magnetized matter fluctuations evolve free of spatial curvature effects and (7.2.4) is no longer required. Note that $c_{\mathrm{a}}^{2} \mathcal{B}=a^{2} \mathrm{D}^{2} B^{2} / \rho$ by definition and therefore the variable $c_{\mathrm{a}}^{2} \mathcal{B}$ is still linear. 


$$
\begin{aligned}
\dot{\Delta}^{(k)}= & \frac{1}{2}\left(1-\frac{1}{2} \beta\right) t^{-1} \Delta^{(k)}-\frac{4}{3} \mathcal{Z}^{(k)}-\frac{1}{2} \beta t^{-1} \mathcal{K}^{(k)}+\frac{3}{4} \beta t^{-1} \mathcal{B}^{(k)}, \\
\dot{\mathcal{Z}}^{(k)}= & -\left(1+\frac{1}{2} \beta\right) t^{-1} \mathcal{Z}^{(k)}-\frac{3}{8}(1-\beta) t^{-2} \Delta^{(k)}-\frac{3}{8} \beta t^{-2} \mathcal{K}^{(k)}+\frac{3}{16} \beta t^{-2} \mathcal{B}^{(k)} \\
& +\frac{1}{4}\left(\frac{k}{a}\right)^{2}\left(1-\frac{1}{2} \beta\right) \Delta^{(k)}+\frac{3}{8}\left(\frac{k}{a}\right)^{2} \beta \mathcal{B}^{(k)}, \\
\dot{\mathcal{K}}^{(k)}= & -\frac{1}{2} \beta t^{-1} \mathcal{K}^{(k)}+\frac{1}{2}\left(1-\frac{1}{2} \beta\right) t^{-1} \Delta^{(k)}+\frac{3}{4} \beta t^{-1} \mathcal{B}^{(k)}, \\
\dot{\mathcal{B}}^{(k)}= & \dot{\Delta}^{(k)} .
\end{aligned}
$$

During the radiation era the Hubble radius, in comoving proper time $t$, is given by $\lambda_{H} \equiv$ $1 / H=2 t$. Also, physical wavelengths and comoving wave numbers are related by $\lambda_{k}=a / k$. Substituting these expressions into Eq. (7.3.3), and keeping up to $\beta$-order terms (recall that $\beta \ll 1)$, the system (7.3.2)-(7.3.5) becomes

$$
\begin{aligned}
\dot{\Delta}^{(k)}= & \frac{1}{2}\left(1-\frac{1}{2} \beta\right) t^{-1} \Delta^{(k)}-\frac{4}{3} \mathcal{Z}^{(k)}-\frac{1}{2} \beta t^{-1} \mathcal{K}^{(k)}+\frac{3}{4} \beta t^{-1} \mathcal{B}^{(k)} \\
\dot{\mathcal{Z}}^{(k)}= & -\left(1+\frac{1}{2} \beta\right) t^{-1} \mathcal{Z}^{(k)}-\frac{3}{8}(1-\beta) t^{-2}\left[1-\frac{1}{6}\left(\frac{\lambda_{H}}{\lambda_{k}}\right)^{2}\left(1+\frac{1}{2} \beta\right)\right] \Delta^{(k)} \\
& -\frac{3}{8} \beta t^{-2} \mathcal{K}^{(k)}+\frac{3}{16} \beta t^{-2}\left[1+\frac{1}{2}\left(\frac{\lambda_{H}}{\lambda_{k}}\right)^{2}\right] \mathcal{B}^{(k)}, \\
\dot{\mathcal{K}}^{(k)}= & -\frac{1}{2} \beta t^{-1} \mathcal{K}^{(k)}+\frac{1}{2}\left(1-\frac{1}{2} \beta\right) t^{-1} \Delta^{(k)}+\frac{3}{4} \beta t^{-1} \mathcal{B}^{(k)}, \\
\dot{\mathcal{B}}^{(k)}= & \dot{\Delta}^{(k)}
\end{aligned}
$$

where $\lambda_{H} / \lambda_{k}=k / a H$. The above describe the evolution of linear matter perturbations in a weakly magnetic almost-FRW universe filled with a single highly conducting perfect fluid.

\subsubsection{Super-horizon scales}

When dealing with perturbations on super-Hubble lengths we have $\lambda_{k} \gg \lambda_{H}$. Then, given also that $\beta \ll 1$, Eqs. (7.3.6)-(7.3.9) reduce to the scale-independent system

$$
\begin{aligned}
\dot{\Delta} & =\frac{1}{2}\left(1-\frac{1}{2} \beta\right) t^{-1} \Delta-\frac{4}{3} \mathcal{Z}-\frac{1}{2} \beta t^{-1} \mathcal{K}+\frac{3}{4} \beta t^{-1} \mathcal{B} \\
\dot{\mathcal{Z}} & =-\left(1+\frac{1}{2} \beta\right) t^{-1} \mathcal{Z}-\frac{3}{8}(1-\beta) t^{-2} \Delta-\frac{3}{8} \beta t^{-2} \mathcal{K}+\frac{3}{16} \beta t^{-2} \mathcal{B}, \\
\dot{\mathcal{K}} & =-\frac{1}{2} \beta t^{-1} \mathcal{K}+\frac{1}{2}\left(1-\frac{1}{2} \beta\right) t^{-1} \Delta+\frac{3}{4} \beta t^{-1} \mathcal{B}, \\
\dot{\mathcal{B}} & =\dot{\Delta}
\end{aligned}
$$


These can be solved analytically and lead to the following simple power-law evolution for the density contrast

$$
\Delta=\Delta(t)=\mathcal{C}_{0}+\mathcal{C}_{i} t^{z_{i}}
$$

where $\mathcal{C}_{0}, \mathcal{C}_{i}$ are constants (with $i=1,2,3$ ) and the $z_{i}$ are roots of a cubic algebraic equation. Ignoring terms of order $\mathcal{O}\left(\beta^{2}\right)$ and higher, the latter reads

$$
8 z^{3}-4(1-\beta) z^{2}-4(1-\beta) z=4 \beta
$$

At the weak-field limit the above is solved perturbatively giving $z_{1}=-1 / 2+5 \beta / 6, z_{2}=-\beta$ and $z_{3}=1-\beta / 3$, which are all physically allowed solutions. Consequently, at this level of approximation, the linear evolution of $\Delta$ proceeds as a sum of power-laws:

$$
\Delta=\mathcal{C}_{0}+\mathcal{C}_{1} t^{-\frac{1}{2}+\frac{5}{6} \beta}+\mathcal{C}_{2} t^{-\beta}+\mathcal{C}_{3} t^{1-\frac{1}{3} \beta}
$$

Note that in the absence of the magnetic field (i.e. for $\beta=0$ ), we recover the standard evolution for the density contrast, familiar from the linear study of perturbed magnetic-free FRW models (e.g. see [155]-[157]). Thus, in the weak-field limit the main magnetic effect is to reduce the growth rate of the dominant density mode. In addition, the field also decreases the rate of the standard decay mode and introduces a new 'non-adiabatic' decay mode 10

\subsubsection{Sub-horizon scales}

Well below the horizon scale, $\lambda_{H} / \lambda_{k} \gg 1$ and the scale-dependent terms inside the brackets of Eq. (7.3.7) become important. Then, on sub-horizon scales the system (7.3.6)-(7.3.9) reads

$$
\begin{aligned}
\dot{\Delta}^{(k)}= & \frac{1}{2}\left(1-\frac{1}{2} \beta\right) t^{-1} \Delta^{(k)}-\frac{4}{3} \mathcal{Z}^{(k)}-\frac{1}{2} \beta t^{-1} \mathcal{K}^{(k)}+\frac{3}{4} \beta t^{-1} \mathcal{B}^{(k)}, \\
\dot{\mathcal{Z}}^{(k)}= & -\left(1+\frac{1}{2} \beta\right) t^{-1} \mathcal{Z}^{(k)}+\frac{1}{16}\left(\frac{\lambda_{H}}{\lambda_{k}}\right)_{0}^{2}\left(1-\frac{1}{2} \beta\right) t_{0}^{-1} t^{-1} \Delta^{(k)} \\
& -\frac{3}{8} \beta t^{-2} \mathcal{K}^{(k)}+\frac{3}{32} \beta\left(\frac{\lambda_{H}}{\lambda_{k}}\right)_{0}^{2} t_{0}^{-1} t^{-1} \mathcal{B}^{(k)} \\
\dot{\mathcal{K}}^{(k)}= & -\frac{1}{2} \beta t^{-1} \mathcal{K}^{(k)}+\frac{1}{2}\left(1-\frac{1}{2} \beta\right) t^{-1} \Delta^{(k)}+\frac{3}{4} \beta t^{-1} \mathcal{B}^{(k)} \\
\dot{\mathcal{B}}^{(k)}= & \dot{\Delta}^{(k)}
\end{aligned}
$$

given that during the radiation era $\lambda_{H} / \lambda_{k}=\left(\lambda_{H} / \lambda_{k}\right)_{0}\left(t / t_{0}\right)^{1 / 2}$.

\footnotetext{
$\overline{10}$ The magnetic suppression of the growing $\Delta$-mode was first observed in [100], although there the 3 -curvature effects were switched off. Allowing for the curvature effects, the analysis of [101] showed no magnetic effect on the growing mode to lowest order in $\beta$, but a slight magnetic enhancement at higher order (see solution (54), (55) there). Here, the refined solutions showed a suppression proportional to $\beta$ in line with the results of [100].
} 
When the 3-curvature effects are switched off, as is physically appropriate during the early universe, the system can be solved analytically. In particular, the solution for the linear matter perturbations reads

$$
\Delta^{(k)}=\Delta^{(k)}(t)=\mathcal{C}_{1}+\mathcal{C}_{2} t^{1 / 4} \mathrm{~J}_{1 / 2+\beta}(\chi)+\mathcal{C}_{3} t^{1 / 4} \mathrm{Y}_{1 / 2+\beta}(\chi)
$$

where $\mathcal{C}_{i}$ are the integration constants (with $\left.i=1,2,3\right)$ and $\mathrm{J}_{1 / 2+\beta}(\chi)$ and $\mathrm{Y}_{1 / 2+\beta}(\chi)$ are Bessel functions of the first and second kind respectively, with arguments determined by

$$
\chi=c_{s}\left(\frac{\lambda_{H}}{\lambda_{k}}\right)\left(1+\frac{1}{2} \beta\right)=c_{s}\left(\frac{\lambda_{H}}{\lambda_{k}}\right)_{0}\left(\frac{t}{t_{0}}\right)^{1 / 2}\left(1+\frac{1}{2} \beta\right),
$$

and $c_{s}=1 / \sqrt{3}$. Also, recalling that $\beta \ll 1$ and keeping up to $\beta$-order terms, we arrive at the following large-scale solution for $\mathcal{B}$ :

$$
\mathcal{B}^{(k)}=\mathcal{B}^{(k)}(t)=-\frac{2}{3} \mathcal{C}_{1}\left(1-\frac{1}{2} \beta\right) \beta^{-1}+\mathcal{C}_{2} t^{1 / 4} \mathrm{~J}_{1 / 2+\beta}(\chi)+\mathcal{C}_{3} t^{1 / 4} \mathrm{Y}_{1 / 2+\beta}(\chi)
$$

As expected (see Eq. (7.3.20) $)$, the above is identical to (7.3.21) up to a constant. Setting $\Delta^{(k)}=\Delta_{0}^{(k)}$ and $\mathcal{B}^{(k)}=\mathcal{B}_{0}^{(k)}$ initially, solutions (7.3.21) and (7.3.23) combine to give $\mathcal{C}_{1} \simeq$ $3 \beta\left(\Delta_{0}^{(k)}-\mathcal{B}_{0}^{(k)}\right) / 2$. Then,

$$
\Delta^{(k)}=\frac{3}{2} \beta\left(\Delta_{0}^{(k)}-\mathcal{B}_{0}^{(k)}\right)+\mathcal{C}_{2} t^{1 / 4} \mathrm{~J}_{1 / 2+\beta}(\chi)+\mathcal{C}_{3} t^{1 / 4} \mathrm{Y}_{1 / 2+\beta}(\chi) .
$$

Based on the weakness of the magnetic field, we may approximate the two Bessel functions in the above given solutions by $J_{1 / 2+\beta} \simeq \mathrm{J}_{1 / 2}$ and $\mathrm{Y}_{1 / 2+\beta} \simeq \mathrm{Y}_{1 / 2}$ respectively. In this case (7.3.24) reduces to

$$
\Delta^{(k)}=\frac{3}{2} \beta\left(\Delta_{0}^{(k)}-\mathcal{B}_{0}^{(k)}\right)+\mathcal{C}_{2} \sqrt{\frac{2}{\pi \alpha}} \sin \left(\alpha t^{1 / 2}\right)+\mathcal{C}_{3} \sqrt{\frac{2}{\pi \alpha}} \cos \left(\alpha t^{1 / 2}\right),
$$

where $\alpha=c_{s}\left(\lambda_{H} / \lambda_{k}\right)_{0} t_{0}^{-1 / 2}(1+\beta / 2)$. Therefore, we find that small-scale matter perturbations oscillate like magneto-sonic waves. The magnetic presence tends to reduce the amplitude of the oscillation and increase its frequency (in agreement with [100,101]). In both cases the effect of the field is proportional to its relative strength (i.e. to the ratio $\beta=B^{2} / \rho$ ). As pointed out in 63], the increased frequency of $\Delta^{(k)}$ should bring the peaks of short-wavelength oscillations in the density of the radiation component closer. This in turn could produce a potentially observable signature in the CMB.

An additional magnetic effect arises from the presence of a constant mode in solution (7.3.25). This suggests that, unlike the magnetic-free case (e.g. see [156]), magnetic matter perturbations in the pre-equality universe oscillate around a generally non-zero average value. This depends on the relation between $\Delta$ and $\mathcal{B}$ initially. For example, isocurvature initial conditions typically correspond to zero perturbation in the total energy density [165,167]. In our case $\rho_{t}=\rho+B^{2} / 2$ and the isocurvature requirement translates into $\Delta_{0}=(\beta / 2) \mathcal{B}_{0}$ (recall 
that $\beta=$ constant throughout this epoch). Following (7.3.25), the latter implies that by the end of the radiation era the associated density contrast oscillates around the average value $\langle\Delta\rangle \simeq 3 \Delta_{0}$. We finally note that, strictly speaking, the analysis of $\S[.3$ holds as long as the electrons are still relativistic (i.e. up to $T \sim T_{e}$ ). Subsequently, and until the end of the radiation era, one should examine the magnetic effects on perturbed non-relativistic matter on an expanding radiative background.

\subsection{Evolution in the dust era}

After the end of the radiation era, the unperturbed background is well approximated by $w=0=c_{s}^{2}, H=2 / 3 t, \rho=4 / 3 t^{2}$. Also, $c_{\mathrm{a}}^{2}=\beta$ and it is no longer constant but decreases with time according to $\beta \propto a^{-1} \propto t^{-2 / 3}$. Applying the usual harmonic decomposition to the perturbation variables, and keeping up to $\beta$-order terms, the system (7.2.1)-(7.2.4) reads

$$
\begin{aligned}
\dot{\Delta}^{(k)}= & -\mathcal{Z}^{(k)}-\frac{2}{3} \beta t^{-1} \mathcal{K}^{(k)}+\beta t^{-1} \mathcal{B}^{(k)} \\
\dot{\mathcal{Z}}^{(k)}= & -\frac{4}{3}\left(1+\frac{2}{3} \beta\right) t^{-1} \mathcal{Z}^{(k)}-\frac{2}{3}\left(1-\frac{4}{3} \beta\right) t^{-2} \Delta^{(k)}-\frac{2}{3} \beta t^{-2} \mathcal{K}^{(k)} \\
& +\frac{1}{3} \beta\left[1+\frac{2}{3}\left(\frac{\lambda_{H}}{\lambda_{k}}\right)^{2}\right] t^{-2} \mathcal{B}^{(k)}, \\
\dot{\mathcal{K}}^{(k)}= & -\frac{8}{9} \beta t^{-1} \mathcal{K}^{(k)}+\frac{4}{3} \beta t^{-1} \mathcal{B}^{(k)}, \\
\dot{\mathcal{B}}^{(k)}= & \frac{4}{3} \dot{\Delta}^{(k)},
\end{aligned}
$$

with $\lambda_{H}=3 t / 2$. The above system has no simple analytical solution because during the dust era the dimensionless parameter $\beta$ is no longer constant. Nevertheless, as we shall see next, one can still extract useful qualitative and quantitative information about the magnetic effects on density perturbations in the post-recombination universe.

\subsubsection{Magnetically-induced Jeans' length}

The wave equation of the density contrast reveals the role of the magnetic pressure against gravitational collapse and helps to establish the scale of the magnetic Jeans' length. In par-

ticular, taking the time derivative of (7.4.1) and using the rest of the formulae we arrive at

$$
\begin{aligned}
\ddot{\Delta}^{(k)}= & -\frac{4}{3}\left(1-\frac{1}{3} \beta\right) t^{-1} \dot{\Delta}^{(k)}+\frac{2}{3}\left\{1-\frac{8}{3} \beta\left[1+\frac{1}{6}\left(\frac{\lambda_{H}}{\lambda_{k}}\right)^{2}\right]\right\} t^{-2} \Delta^{(k)} \\
& +\frac{8}{9} \beta t^{-2} \mathcal{K}^{(k)} .
\end{aligned}
$$


Note that, in deriving the above, we have set $\mathcal{B}_{0}^{(k)}=4 \Delta_{0}^{(k)} / 3$. This guarantees that $\mathcal{B}^{(k)}=$ $4 \Delta^{(k)} / 3$ always (see Eq. (17.4.4)).

The first term on the right-hand side of (7.4.5) shows that the magnetic presence reduces the diluting effect of the expansion by a small amount proportional to the field's relative strength. The second term gauges the opposing effects of gravity and magnetic pressure. When the curvature term is removed form (7.4.5), and the quantity in the angled brackets is positive, gravity prevails and the density contrast grows. When the brackets take a negative value, the pressure of the magnetic field dominates and forces the inhomogeneity into an oscillatory phase. In the latter case the magnetic effect is relatively weak and also decays in time. Gravitational attraction and magnetic pressure balance each other out at the threshold $1-(8 \beta / 3)\left[1+\left(\lambda_{H} / \lambda_{k}\right)^{2} / 6\right]=0$. This also determines an associated Jeans length, defined as the scale below which the magnetic pressure gradients dominate and the inhomogeneities oscillate. This 'magnetic' Jeans length is given by

$$
\lambda_{J}=\sqrt{\frac{4 \beta}{9-24 \beta}} \lambda_{H}
$$

and it is considerably smaller than the corresponding Hubble radius since $\beta \ll 1$ (see also [92,93] for analogous expressions). The anisotropy of the CMB constrains the current magnitude of a (homogeneous) cosmological magnetic field below $10^{-9} G$ 63,66. In that case $B_{0}^{2} \sim$ $10^{-58} \mathrm{GeV}^{4}, \beta_{0} \sim 10^{-11}$ (recall that $\rho=\rho_{\mathrm{cr}} \sim 10^{-47} \mathrm{GeV}^{4}$ today) and the associated magnetic Jeans length is

$$
\lambda_{J} \sim 10^{-5} \lambda_{H} \sim 10 \mathrm{Kpc}
$$

today. Random $B$-fields, however, can have magnitudes up to $10^{-6} G$ in today's values. If $B_{0} \sim 10^{-7} G$, a strength supported by observations in galaxy clusters and high-redshift protogalactic structures, we find that

$$
\lambda_{J} \sim 10^{-3} \lambda_{H} \sim 1 \mathrm{Mpc}
$$

at present. This number, which can also be obtained through a fully Newtonian treatment [95], determines the dimensions of the region that is gravitationally supported by the magnetic pressure gradients, and lies intriguingly close to the size of a galaxy cluster.

\subsubsection{Late-time evolution}

One can obtain an analytical solution for the evolution of magnetic density perturbations in the dust era by taking the time derivative of (7.4.5). Then, recalling that $\lambda_{H} / \lambda_{k}=$ $\left(\lambda_{H} / \lambda_{k}\right)_{0}\left(t / t_{0}\right)^{1 / 3}$, using (7.4.3) and (7.4.4), and keeping up to $\beta$-order terms we arrive at 


$$
\begin{aligned}
\dddot{\Delta}^{(k)}= & -4\left[1-\frac{1}{9} \beta_{0}\left(\frac{t_{0}}{t}\right)^{2 / 3}\right] t^{-1} \ddot{\Delta}^{(k)}-\frac{14}{9}\left[1+\frac{4}{21} \beta_{0}\left(\frac{\lambda_{H}}{\lambda_{k}}\right)_{0}^{2}+\frac{6}{7} \beta_{0}\left(\frac{t_{0}}{t}\right)^{2 / 3}\right] t^{-2} \dot{\Delta}^{(k)} \\
& +\frac{4}{9}\left[1-\frac{4}{9} \beta_{0}\left(\frac{\lambda_{H}}{\lambda_{k}}\right)_{0}^{2}\right] t^{-3} \Delta^{(k)}+\mathcal{C}_{\mathcal{B}} t^{-3} .
\end{aligned}
$$

The constant $\mathcal{C}_{\mathcal{B}}$ is inversely proportional to the scale in question and vanishes when $\mathcal{B}_{0}=$ $4 \Delta_{0} / 3$. As we will see in $\S 9$, the aforementioned initial conditions guarantee effective adiabaticity. Ignoring the weak and time-decaying terms from the right-hand side of (17.4.9), we obtain the following late-time solution (see also Eq. (67) in [101])

$$
\Delta^{(k)}=\mathcal{C}_{1} t^{\alpha_{1}}+\mathcal{C}_{2} t^{\alpha_{2}}+\mathcal{C}_{3} t^{-2 / 3}+\mathcal{C}_{4}
$$

with

$$
\alpha_{1,2}=-\frac{1}{6}\left[1 \pm 5 \sqrt{1-\frac{32}{75} \beta_{0}\left(\frac{\lambda_{H}}{\lambda_{k}}\right)_{0}^{2}}\right] .
$$

In the absence of the $B$-field (i.e. when $\beta_{0}=0$ ), we immediately recover the standard magneticfree solution with $\alpha_{1}=2 / 3$ and $\alpha_{2}=-1$ (e.g. see [155]-[157]). Therefore, the main magnetic effect is to reduce the growth rate of density perturbations by an amount proportional to its relative strength (i.e. to the ratio $\left.\beta_{0}=\left(B^{2} / \rho\right)_{0}\right)$. It should be noted that the inhibiting role of the field was first observed in the Newtonian treatment of [90] and later in the relativistic studies of [100,101]. According to (7.4.10) and (7.4.11), the magnetic impact is inversely proportional to the scale in question. On super-horizon lengths, in particular, the above given solution reads

$$
\Delta^{(k)}=\mathcal{C}_{1} t^{2 / 3}+\mathcal{C}_{2} t^{-1}+\mathcal{C}_{3} t^{-2 / 3}
$$

since $\mathcal{C}_{4} \simeq\left(\beta_{0} / 3\right)\left(\lambda_{H} / \lambda_{k}\right)_{0}^{2}\left[\mathcal{B}_{0}-(4 / 3) \Delta_{0}\right] \ll 1$. Hence, on large scales the introduction of the $B$-field simply adds the decaying $t^{-2 / 3}$ mode to the standard magnetic-free result. Note also that $\Delta$ describes the directionally-averaged gravitational clumping of the matter. Generally, the perturbations will grow at different rates parallel and perpendicular to the magnetic field and so there will also be non-spherical evolution in the shapes of these distortions.

\subsection{Evolution in false-vacuum environments}

False vacuum cosmological environments have been largely associated with inflation, since they typically lead to exponential, de Sitter-type expansion. Dynamically, such environments can be achieved by introducing a fluid with a non-conventional equation of state. Here, we will consider linear inhomogeneities in the energy density of a barotropic and of a non-barotropic magnetized medium with a false-vacuum equation of state.

Nonlinear spatial inhomogeneities in the density distribution of a perfect, though not neces- 
sarily barotropic, fluid propagate according to 11

$$
\dot{\Delta}_{\langle a\rangle}=\frac{3 p}{\rho} H \Delta_{a}-\frac{3 a H}{\rho} \mathrm{D}_{a} p-(\rho+p) \mathcal{Z}_{a}-3 a(\rho+p) H A_{a}-\left(\sigma_{b a}+\omega_{b a}\right) \Delta^{b},
$$

The 4-acceleration vector seen above satisfies the momentum-density conservation law. In the absence of a magnetic field the latter reads

$$
(\rho+p) A_{a}=-\mathrm{D}_{a} p
$$

with $\rho+p$ representing the inertial mass of the fluid. When dealing with a magnetized medium, however, (7.5.2) is replaced by

$$
\left(\rho+p+\frac{2}{3} B^{2}\right) A_{a}=-\mathrm{D}_{a} p-\varepsilon_{a b c} B^{b} \operatorname{curl} B^{c}-\Pi_{a b} A^{b}
$$

which incorporates the magnetic contribution to the total inertial mass of the system.

One way of achieving the false vacuum environment is by introducing an effective (barotropic) perfect fluid with $p=-\rho$. In that case Eq. (7.5.2) acts as a constraint demanding that $\mathrm{D}_{a} p=0$. This immediately implies $\mathrm{D}_{a} \rho=0$ and therefore zero density inhomogeneities. This strict constraint is a direct and unavoidable consequence of having a medium with zero inertial mass. When a magnetic field is present, however, the 4-acceleration is given by (7.5.3) and this expression allows for nonzero pressure gradients even if $\rho+p=0$. In that case we have $\mathrm{D}_{a} p=-\mathrm{D}_{a} \rho \neq 0$ and Eq. (7.5.1) reduces to

$$
\dot{\Delta}_{a}=0
$$

at the linear perturbative level. Accordingly, all three types of linear density inhomogeneities (i.e. matter perturbations, vortices and shape distortions) remain constant as long as $\rho+p=0$. Note that we always assume that $B^{2} \ll \rho$, which ensures that the magnetic field has no effect of the exponential expansion of the model (i.e. $\rho+3 p+B^{2} \simeq \rho+3 p=-2 \rho<0$ ).

Standard de Sitter inflation is typically achieved by means of a single minimally coupled scalar field $(\varphi)$, which generally does not behave like a barotropic medium. Instead, the $\varphi$-field acts as an effective perfect fluid with energy density and pressure given by

$$
\rho=\rho^{(\varphi)}=\frac{1}{2} \dot{\varphi}^{2}+V(\varphi) \quad \text { and } \quad p=p^{(\varphi)}=\frac{1}{2} \dot{\varphi}^{2}-V(\varphi),
$$

respectively (e.g. see [158,159]). Therefore, the scalar field corresponds to a barotropic fluid only when its kinetic or potential energies vanishes 12 In the first instance we have $\dot{\varphi}=0$ and $p^{(\varphi)}=-\rho^{(\varphi)}=-V(\varphi)$, while in the second Eqs. (7.5.5) give $p^{(\varphi)}=\rho^{(\varphi)}=\dot{\varphi}^{2} / 2$. In any other case $p=\rho-2 V(\varphi)=\dot{\varphi}^{2}-\rho$. The perfect-fluid description of a minimally coupled scalar

\footnotetext{
${ }^{11}$ One can derive (7.5.1) from first principle, namely by taking the proper-time derivative of definition (5.7.1 $)$ ), or directly from (5.7.4) by substituting the 4-acceleration from Eq. (3.3.2).

12 Non-minimally coupled scalar fields generally correspond to imperfect fluids with more complicated expressions for their associated effective density and pressure (e.g. see [160]).
} 
field refers to a family of observers moving with the (timelike) 4-velocity $u_{a}=-\nabla_{a} \varphi / \dot{\varphi}$, where $\nabla_{a} \varphi \neq 0$ and $\dot{\varphi}=u^{a} \nabla_{a} \varphi$. Relative to the same observers, $\mathrm{D}_{a} \varphi=0$ and therefore $\mathrm{D}_{a} p^{(\varphi)}=\mathrm{D}_{a} \rho^{(\varphi)}=\dot{\varphi} \mathrm{D}_{a} \dot{\varphi}$ [159]. This last result indicates a major change relative to the barotropic-fluid case and it will have a profound effect on the evolution of the inhomogeneities.

The de Sitter phase occurs when the scalar field rolls slowly down its potential. Throughout this slow-rolling regime, $\dot{\varphi}^{2} \ll V(\varphi)$ and expressions (7.5.5) imply that $p^{(\varphi)} \simeq-\rho^{(\varphi)} \simeq-V(\varphi)$. In this environment we may, to first approximation, ignore the last three terms in the righthand side of (7.5.1), which then linearizes to

$$
\dot{\Delta}_{a}=-6 H \Delta_{a}
$$

where $H=\dot{a} / a \simeq$ constant and $a \propto \exp (H t)$. This result suggests an exponential decay for all three types of density inhomogeneities irrespective of the magnetic presence and in line with the cosmic no-hair theorems [161]-[164].

We note that here we have only considered the dynamical effect of the exponential expansion on the evolution of perturbations in the presence of the $B$-field. Changes in the electrical properties of the cosmic medium, which can occur during the de Sitter regime, have not been accounted for. Also, typical linear studies, in magnetic-free universes, consider the secondtime derivative of the density gradients instead of the first. This means that one decouples the expansion gradients from (7.5.1) before setting $\rho+p \simeq 0$. One could do the same in the magnetic case as well. However, the calculation is rather involved and goes beyond the scope of this section. We expect that the inhomogeneities will still be found to decay exponentially, though probably at a slightly slower rate.

\section{Isocurvature perturbations}

One can define as isocurvature perturbations those occurring on hypersurfaces of uniform curvature, namely fluctuations which maintain $\mathrm{D}_{a} \mathcal{R}=0$ at all times [147]. This should be distinguished from the definition typically found in the literature, where the term isocurvature means distortions in multi-component systems with zero perturbation in the total energydensity initially (e.g. see [165167]). Here we will impose the $\mathrm{D}_{a} \mathcal{R}=0$ condition throughout the evolution of the perturbed mode, noting that one could use the equations given in section $\S 7$ to study fluctuations with an isocurvature initial condition by setting $\Delta_{0}+\left(\beta_{0} / 2\right) \mathcal{B}_{0}=0$ (see $\S 7.3 .2)$.

\subsection{Consistency condition}

Isocurvature perturbations also require zero vorticity to guarantee the integrability of the 3-D hypersurfaces orthogonal to $u_{a}$. In the magnetic presence, linear vortices are switched off by imposing the (self-consistent) constraint $B^{b} \mathrm{D}_{b} \operatorname{curl} B_{a}=0$ (see Eq. (6.2.4)). The zero-curvature 
condition for isocurvature magnetic perturbations is obtained through the orthogonally projected gradient of ([6.2.9). To be precise, using definitions (5.7.1), (5.7.2 a) and linearising around our FRW background we arrive at

$$
a \mathrm{D}_{a} \mathcal{R}=2 \rho \Delta_{a}+c_{\mathrm{a}}^{2} \rho(1+w) \mathcal{B}_{a}-4 H \mathcal{Z}_{a} .
$$

When $\mathrm{D}_{a} \mathcal{R}=0$, the right-hand side of the above ensures that linear expansion gradients and those in the fluid and the magnetic energy densities are connected by a simple algebraic relation. The projected comoving divergence of the latter translates into the following linear constraint between the associated scalar variables

$$
2 H \mathcal{Z}=\rho\left[\Delta+\frac{1}{2} c_{\mathrm{a}}^{2}(1+w) \mathcal{B}\right]
$$

In the absence of the magnetic field, this condition is automatically satisfied for pressure-free dust, but holds on large scales only when the matter has non-zero pressure [147]. If a magnetic field is present the vanishing of the matter pressure is not enough to guarantee that $\mathrm{D}_{a} \mathcal{R}=0$ at all times, because of the Lorentz-force contribution to the 4 -acceleration. In that case the consistency condition for linear isocurvature perturbations is satisfied on large scales only. Indeed, on using the linearized propagation equation of the spatial Ricci scalar, we arrive at

$$
\left(\mathrm{D}_{a} \mathcal{R}\right)^{\cdot}=-3\left(1+\frac{4}{9} c_{\mathrm{a}}^{2}\right) H \mathrm{D}_{a} \mathcal{R}+\frac{4 c_{s}^{2} H}{a(1+w)}\left(1-\frac{2}{3} c_{\mathrm{a}}^{2}\right) \mathrm{D}^{2} \Delta_{a}+\frac{2 c_{\mathrm{a}}^{2} H}{a} \mathrm{D}^{2} \mathcal{B}_{a}
$$

since the vorticity has already been switched off. This result shows that the linear isocurvature condition is self-maintained only asymptotically (i.e. at infinity) where the Laplacians of the right-hand side vanish. Nevertheless, following [147]-[149], we will assume that on sufficiently long wavelengths the source terms in the right-hand side of (8.1.3) are negligible. Note the last term in the right-hand side of the above, which demonstrates why zero fluid pressure does not automatically guarantee the consistency of $\mathrm{D}_{a} \mathcal{R}=0$ in the presence of the $B$-field.

Using the isocurvature condition (8.1.2), we can eliminate $\mathcal{Z}$ from Eq. (7.2.1), which reduces to

$$
\dot{\Delta}=-\frac{3}{2}\left(1-w+\frac{4}{3} c_{\mathrm{a}}^{2} w\right) H \Delta+\frac{3}{4} c_{\mathrm{a}}^{2}\left(1-w^{2}\right) H \mathcal{B}-c_{\mathrm{a}}^{2}(1+w) H \mathcal{K} .
$$

The above, together with (77.2.3) and (7.2.4), describes the linear evolution of isocurvature scalar perturbations on a weakly magnetic flat FRW background filled with a single highly conducting perfect fluid.

\subsection{Evolution in the radiation era}

Since isocurvature perturbations apply to super-horizon scales only, all gradients in the fluid and the magnetic pressure have been switched off. This means that the only support against the gravitational pull of the matter comes from the expansion of the universe. When relativistic matter dominates the energy density of the latter, the system (8.1.4), (7.2.3) and (7.2.4) reduces to 


$$
\begin{aligned}
\dot{\Delta} & =-\frac{1}{2}\left(1+\frac{1}{2} \beta\right) t^{-1} \Delta-\frac{1}{2} \beta t^{-1} \mathcal{K}+\frac{1}{4} \beta t^{-1} \mathcal{B}, \\
\dot{\mathcal{K}} & =-\frac{1}{2} \beta t^{-1} \mathcal{K}+\frac{1}{2}\left(1-\frac{1}{2} \beta\right) t^{-1} \Delta+\frac{3}{4} \beta t^{-1} \mathcal{B}, \\
\dot{\mathcal{B}} & =\dot{\Delta} .
\end{aligned}
$$

Taking into account that $\beta=$ constant $\ll 1$ and keeping up to $\beta$-order terms, the solution for magnetised isocurvature perturbations reads

$$
\Delta=\frac{1}{2} \beta\left(\Delta_{0}-\mathcal{B}_{0}\right)+\mathcal{C}_{1} t^{-\beta}+\mathcal{C}_{2} t^{-(1-\beta) / 2}
$$

where $\Delta_{0}, \mathcal{B}_{0}$ are the perturbed matter and magnetic energy densities initially and $\mathcal{C}_{1,2}$ are constants (see also [101]). When $\Delta_{0} \neq \mathcal{B}_{0}$, the isocurvature density contrast approaches a non-zero value which depends on the initial conditions.

\subsection{Evolution in the dust era}

After matter-radiation equality, the system (8.1.4), (7.2.3) and (7.2.4) becomes

$$
\begin{aligned}
\dot{\Delta} & =-t^{-1} \Delta-\frac{2}{3} \beta t^{-1} \mathcal{K}+\frac{1}{2} \beta t^{-1} \mathcal{B}, \\
\dot{\mathcal{K}} & =-\frac{8}{9} \beta t^{-1} \mathcal{K}+\frac{4}{3} \beta t^{-1} \mathcal{B}, \\
\dot{\mathcal{B}} & =\frac{4}{3} \dot{\Delta}
\end{aligned}
$$

with $\beta=\beta_{0}\left(t_{0} / t\right)^{2 / 3}$ throughout the dust era. Because of this time-variation in the effective sound speed, the above system has no simple analytical solution. Nevertheless, taking the time derivative of (8.3.1), using (8.3.2), (8.3.3) and keeping up to $\beta_{0}$-order terms we arrive at

$$
\ddot{\Delta}=-\frac{8}{3}\left[1-\frac{1}{4} \beta_{0}\left(\frac{t_{0}}{t}\right)^{2 / 3}\right] t^{-1} \dot{\Delta}-\frac{2}{3} t^{-2} \Delta .
$$

At late times, we may ignore the weak and decaying $\beta_{0}\left(t_{0} / t\right)^{2 / 3}$ term in the right-hand side of the above, which then admits the power-law solution [101]

$$
\Delta=\mathcal{C}_{1} t^{-2 / 3}+\mathcal{C}_{2} t^{-1}
$$

Comparing results (8.2.4) and (8.3.5) to their magnetic-free counterparts, shows that the presence of the $B$-fieldd has only added new decaying isocurvature modes. Recall that in single-fluid almost-FRW cosmologies, isocurvature modes evolve as $\Delta \propto t^{-1 / 2}$ during the radiation era and like $\Delta \propto t^{-1}$ after equality [147]. 


\section{$9 \quad$ Entropy perturbations}

\subsection{Entropy perturbations in multi-component systems}

Entropy perturbations can and will generically arise in all multi-component systems. The magnetized, highly conductive barotropic medium may be treated as a mixture of two comoving fluids, of which one (the matter component) is perfect and the other (the $B$-field) is imperfect (see Eq. (5.3.1) ). Then, it would be interesting to see whether some of the magnetic effects can be interpreted as coming from an effective entropy perturbation caused by differences in the dynamical behaviour of the two fluids.

Assuming that $\mathcal{E}_{a}^{(i)}$ is the intrinsic entropy perturbation associated with the $i$-th component of a multi-fluid system, the effective entropy perturbation of the total fluid is given by the expression [125]-[127]

$$
\mathcal{E}_{a}=\frac{1}{\bar{p}} \sum_{i} p^{(i)} \mathcal{E}_{a}^{(i)}+\frac{1}{2 \bar{p} \bar{h}} \sum_{i j} h^{(i)} h^{(j)}\left(c_{s}^{2(i)}-c_{s}^{2(j)}\right) \mathcal{S}_{a}^{(i j)} .
$$

Here, $h^{(i)}=\rho^{(i)}+p^{(i)}$ represents the inertial mass of the species, while $\bar{p}=\sum_{i} p^{(i)}$ and $\bar{h}=\sum_{i} h^{(i)}$ are respectively the isotropic pressure and the inertial mass of the mixture. Also, the orthogonally projected vector

$$
\mathcal{S}_{a}^{(i j)}=\frac{\rho^{(i)}}{h^{(i)}} \Delta_{a}^{(i)}-\frac{\rho^{(j)}}{h^{(j)}} \Delta_{a}^{(j)}
$$

with $\Delta_{a}^{(i)}=\left(a / \rho^{(i)}\right) \mathrm{D}_{a} \rho^{(i)}$ and $\mathcal{S}_{a}^{(i j)}=-\mathcal{S}_{a}^{(j i)}$, describes the effective entropy perturbation triggered by the different dynamical behaviour of the individual components. Therefore, in a multi-fluid system, the total entropy fluctuation has one part coming from the intrinsic entropy perturbation of each species and one coming from differences in their dynamics. In this sense, a state of effective overall adiabaticity corresponds to $\mathcal{E}_{a}=0$. Note that, according to (9.1.1), there is no entropy perturbation coming from differences in the dynamics of the member species when these share the same effective sound speed.

\subsection{Magnetically-induced entropy perturbations}

Treating the highly conducting magnetized medium as a system of two comoving fluids, we may only consider entropy perturbations due to the different dynamical evolution of the two components. These are determined by

$$
\mathcal{S}_{a}^{(12)}=\frac{1}{1+w} \Delta_{a}-\frac{3}{4} \mathcal{B}_{a}
$$

given that $\rho^{(1)}=\rho, \rho^{(2)}=B^{2} / 2, h^{(1)}=\rho+p, h^{(2)}=2 B^{2} / 3, c_{s}^{2(1)}=c_{s}^{2}, c_{s}^{2(2)}=1 / 3$ (since $\left.p_{B}=\rho_{B} / 3=B^{2} / 6\right), \Delta_{a}^{(1)}=\Delta_{a}, \Delta_{a}^{(2)}=\mathcal{B}_{a}, \bar{p}=p+B^{2} / 6$ and $\bar{h}=\rho+p+2 B^{2} / 3$. However, linear 
perturbations in the magnetic energy density evolve in step with their matter counterparts (see Eq. (6.3.19) ). This guarantees that

$$
\mathcal{S}_{a}^{(12)}=\frac{3}{4} \mathcal{C}_{a}
$$

where $\mathcal{C}_{a}=\mathcal{B}_{a}^{0}-4 \Delta_{a}^{0} / 3(1+w)$ is a constant that depends on the initial conditions. Substituted into definition (9.1.1), the above gives

$$
\mathcal{E}_{a}=-\frac{3 \rho(1+w)\left(3 c_{s}^{2}-1\right) B^{2}}{\left(6 p+B^{2}\right)\left[3 \rho(1+w)+2 B^{2}\right]} \mathcal{C}_{a}
$$

This vanishes during the radiation era - a result guaranteed by the radiation-like behaviour of the $B$-field (i.e. by the fact that $p_{B}=\rho_{B} / 3$ - see also [166]). In the dust epoch, on the other hand, we find that $\mathcal{E}_{a} \simeq \mathcal{C}_{a}=$ constant (since $B^{2} \ll \rho$ always). These mean that, within the two-fluid description, part of the magnetic effect on the linear evolution of matter inhomogeneities can be interpreted as an effective entropy perturbation, due to the different dynamics of the two perturbed fluids, but only after equality. Then, the value of the effective entropy fluctuation depends on the initial relation between the magnetic and the fluid energydensity perturbations. In particular, when $\mathcal{B}_{a}=4 \Delta_{a} / 3$ initially the dynamically induced entropy perturbation vanishes and we have effective adiabaticity.

\section{Vector perturbations}

A general inhomogeneous perturbation is characterized by its rotational and deformation properties, in addition to the amount of matter aggregation (or dilution). Following [101], we will consider here the effect of magnetic fields on the linear evolution of vortex-like distortions in the density distribution of the cosmic medium.

\subsection{Basic variables}

Rotational instabilities in the fluid density are described by means of the antisymmetric, comoving, orthogonally projected tensor $\mathcal{W}_{a b}=a \mathrm{D}_{[b} \Delta_{a]}$ (see decomposition (6.3.7) in $\S 6.3 .3$ ). The antisymmetry of the latter means that we can use it to define the vector

$$
\mathcal{W}_{a}=\frac{1}{2} \varepsilon_{a b c} \mathcal{W}^{b c}=-\frac{a}{2} \operatorname{curl} \Delta_{a}
$$

with $\mathrm{D}^{a} \mathcal{W}_{a}=0$ to linear order. Recalling that $\Delta_{a}=(a / \rho) \mathrm{D}_{a} \rho$ and using the general relativistic commutation law $\mathrm{D}_{[a} \mathrm{D}_{b]} \phi=-\dot{\phi} \omega_{a b}$ between the projected gradients of scalars (see Eq. (A.1) in $\S$ A.1. , we find that

$$
\mathcal{W}_{a}=-3 a^{2}(1+w) H \omega_{a}
$$


This result reflects a fundamental property of vortex-like distortions in general relativity, namely that they are proportional to the amount of vorticity. Also, (10.1.2) and the linearized version of constraint (2.3.6) guarantee that $\mathcal{W}_{a}$ is a solenoidal vector to first order. Similarly, linear vortices in the volume expansion and the magnetic energy density are also directly related to the vorticity vector and therefore to those in the density of the matter. In particular,

$$
\frac{a}{2} \operatorname{curl} \mathcal{Z}_{a}=\frac{\dot{H}}{(1+w) H} \mathcal{W}_{a} \quad \text { and } \quad \frac{a}{2} \operatorname{curl} \mathcal{B}_{a}=-\frac{4}{3(1+w)} \mathcal{W}_{a}
$$

to first approximation. Finally, combining the antisymmetric part of the linearized GaussCodacci equation (see (5.6.3) in $\S$ 5.6) with Eq. (10.1.2), we obtain

$$
\mathcal{R}_{a}=\frac{1}{3 a^{2}(1+w)} \mathcal{W}_{a}
$$

where $\mathcal{R}_{a}=\varepsilon_{a b c} \mathcal{R}^{b c} / 2$ is the vector component of the local spatial curvature.

\subsection{Linear equations}

Magnetic fields are unique in the sense that they are the only large-scale vector source that has ever been observed in the universe. Given that, the study of vortex-like, vector perturbations in the presence of the $B$-field acquires particular significance. In our analysis, effects directly related to the vector nature of magnetic fields propagate through the tension part of the Lorentz force (see decomposition (5.3.4)). Thus, as far as the scalar/density perturbations are concerned, these effects are encoded in the magneto-curvature terms seen in Eqs. (7.2.1), (7.2.2).

Spatial inhomogeneities in the density distribution of a magnetized and highly conductive barotropic perfect fluid propagate according to the linear expression (6.3.11) in $\S 6.3 .4$. When combined with (6.3.14), the latter takes the form

$$
\begin{aligned}
\dot{\Delta}_{a}= & 3 w\left(1-\frac{2}{3} c_{\mathrm{a}}^{2}\right) H \Delta_{a}-(1+w) \mathcal{Z}_{a}+\frac{3}{2} c_{\mathrm{a}}^{2}(1+w) H \mathcal{B}_{a} \\
& -\frac{3 a H}{\rho}\left(1-\frac{2}{3} c_{\mathrm{a}}^{2}\right) B^{b} \mathrm{D}_{b} B_{a},
\end{aligned}
$$

where we have decomposed the magnetic Lorentz force into its isotropic-pressure and tension parts (see (5.3.4) in $\S 5.3$ ) and kept up to $c_{\mathrm{a}}^{2}$-order terms. Taking the comoving, orthogonally projected gradient of the above and isolating its antisymmetric part we arrive at

$$
\begin{aligned}
\dot{\mathcal{W}}_{a}= & -\frac{3}{2}\left\{1-w-\frac{1}{18} c_{\mathrm{a}}^{2}\left[(1-3 w)^{2}-12 w\right]\right\} H \mathcal{W}_{a} \\
& +\frac{3 a^{2} H}{2 \rho}\left(1-\frac{2}{3} c_{\mathrm{a}}^{2}\right) B^{b} \mathrm{D}_{b} \operatorname{curl} B_{a}
\end{aligned}
$$


to linear order. Note that in deriving this expression we have also used the auxiliary relations (10.1.3), (10.1.4), the commutation laws between the spatial gradients of scalars and orthogonally projected vectors and the zero-order versions of Eqs. (5.5.1), (5.6.4). The above shows that the $B$-field acts as a source of density vortices when $B^{b} \mathrm{D}_{b}$ curl $B_{a} \neq 0$. Not surprisingly, the same requirement can also lead to kinematic vorticity (see Eq. (6.2.4) in $\S$ 6.2). In fact, recalling that $w=c_{s}^{2}=$ constant, one can recover (6.2.4) from (10.2.2) by means of relation (10.1.2). An additional magnetic effect is the precession of $\mathcal{W}_{a}$, since its no longer parallel to $\dot{\mathcal{W}}_{a}$ (see also [101]).

For comparison reasons it will help to consider first the solution of Eq. (10.2.2) in the absence of the $B$-field. Assuming conventional matter and dropping the magnetic terms from the righthand side of the above, it is straightforward to show that non-magnetized fluid vortices decay always and on all scales. In particular, $\mathcal{W}_{a} \propto t^{-1 / 2}$ during the radiation era and $\mathcal{W}_{a} \propto t^{-1}$ after equality. In what follows we will illustrate how the magnetic field changes this picture by focusing on the dust era, referring the reader to [101] for further discussion and details.

\subsection{Evolution in the dust era}

Expression (10.2.2) shows that the presence of the $B$-field will generally trigger vortices in the density distribution of the magnetized matter. Nevertheless, the magnetic effect on preexisting rotational distortions is not clear yet. To quantify the role of the field we need to go one step further and obtain a decoupled equation for the evolution of $\mathcal{W}_{a}$. After equality, the Alfvén speed decays with time according to $c_{\mathrm{a}}^{2}=\beta \propto t^{-2 / 3}$. This means that, very soon, the weak Alfvén terms in the right-hand side of (10.2.2) become completely negligible. At this limit, the time derivative of the latter leads to [101]

$$
\ddot{\mathcal{W}}_{a}=-4 H \dot{\mathcal{W}}_{a}-\frac{1}{2} \rho \mathcal{W}_{a}+\frac{1}{3} c_{\mathrm{a}}^{2} \mathrm{D}^{2} \mathcal{W}_{a}
$$

Thus, during the dust era, magnetized vortices propagate like Alfvén waves with signal speed $v_{\mathrm{a}}=c_{\mathrm{a}} / \sqrt{3}$. Using standard vector harmonics, we may decompose the solenoidal $\mathcal{W}_{a}$ as $\mathcal{W}_{a}=\sum_{k} \mathcal{W}_{(k)} \mathcal{Q}_{a}^{(k)}$, where $\mathrm{D}_{a} \mathcal{W}_{(k)}=0=\dot{\mathcal{Q}}_{a}^{(k)}=\mathrm{D}^{a} \mathcal{Q}_{a}^{(k)}$ and $\mathrm{D}^{2} \mathcal{Q}_{a}^{(k)}=-(k / a)^{2} \mathcal{Q}_{a}^{(k)}$. Then, recalling that $w=0=c_{s}^{2}, a \propto t^{2 / 3}, H=2 / 3 t$ and $\rho=4 / 3 t^{2}$, the $k$-th Fourier mode propagates according to

$$
\ddot{\mathcal{W}}^{(k)}=-\frac{8}{3} t^{-1} \dot{\mathcal{W}}^{(k)}-\frac{2}{3}\left[1+\frac{2}{9}\left(\frac{\lambda_{\mathrm{a}}}{\lambda_{k}}\right)_{0}^{2}\right] t^{-2} \mathcal{W}^{(k)},
$$

with $\lambda_{k}=a / k$ representing the wavelength of the perturbation and $\lambda_{\mathrm{a}}=\sqrt{\beta} \lambda_{H}$. This represents a characteristic scale of the magnetized environment, which may be termed the 'Alfvén horizon' [101]. The above admits the solution

$$
\mathcal{W}^{(k)}=\mathcal{C}_{1} t^{\alpha_{1}}+\mathcal{C}_{2} t^{\alpha_{2}}
$$


where $\mathcal{C}_{1,2}$ are constants and

$$
\alpha_{1,2}=-\frac{1}{6}\left[5 \pm \sqrt{1-\frac{48}{9}\left(\frac{\lambda_{\mathrm{a}}}{\lambda_{k}}\right)_{0}^{2}}\right] .
$$

On scales far beyond the Alfvén horizon, $\lambda_{\mathrm{a}} / \lambda_{k} \ll 1$ and the perturbed mode exhibits a power-law decay with $\mathcal{W} \propto t^{-2 / 3}$, a rate considerably slower than the one associated with dust dominated, magnetic-free cosmologies. Recall that $\mathcal{W} \propto t^{-1}$ in the absence of the $B$-field. Well inside $\lambda_{\mathrm{a}}$, on the other hand, perturbations oscillate like Alfvén waves with a time-decreasing amplitude. In particular, for $\lambda_{\mathrm{a}} / \lambda_{k} \gg 1$, solution (10.3.3), (10.3.4) implies

$$
\mathcal{W}_{(k)} \propto \cos \left[\frac{2 \sqrt{3}}{9}\left(\frac{\lambda_{\mathrm{a}}}{\lambda_{k}}\right)_{0} \ln t\right] t^{-5 / 6} .
$$

Thus, the overall, the effect of the $B$-field on a given vortex mode is to decrease its standard depletion rate.

Not surprisingly, a similar magnetic effect is also observed on vorticity proper. Using the linear relation (10.1.2), we find that after equality large-scale rotational distortions decay as $\omega_{a} \propto t^{-1}$ as opposed to $\omega_{a} \propto t^{-4 / 3}$. On these grounds, magnetized cosmologies would contain more residual vortices than their magnetic-free counterparts.

\section{Gravitational waves}

Gravitational waves are propagating fluctuations in the geometry of the spacetime fabric, usually described as weak perturbations of the background metric. Alternatively, one can describe gravity waves covariantly by means of the electric and magnetic components of the Weyl tensor [168], which encodes the long-range gravitational field, namely the one determined by the presence of matter "elsewhere" in the space (see $\S$ 4.2).

\subsection{Isolating magnetised tensor modes}

Covariantly, gravitational waves are described by the transverse degrees of freedom in the electric $\left(E_{a b}\right)$ and magnetic $\left(H_{a b}\right)$ parts of the conformal curvature tensor. The transversality is necessary to ensure that the pure tensor modes of the locally free gravitational field have been isolated. The same condition is also imposed on the shear and any other orthogonally projected, traceless, second-rank tensor that might be present. Thus, when studying the propagation of gravitational radiation in perturbed FRW models with perfect fluid matter we require that (see $\S 2.3$ and $\S 4.2$ )

$$
\mathrm{D}^{b} E_{a b}=0=\mathrm{D}^{b} H_{a b}=\mathrm{D}^{b} \sigma_{a b}
$$


to linear order and at all times (e.g. see [169]). In magnetic-free universes, this is achieved by switching the vorticity off and by setting $\mathrm{D}_{a} \rho=0=\mathrm{D}_{a} p=\mathrm{D}_{a} \Theta$ (for a barotropic medium it suffices to ensure that $\mathrm{D}_{a} \rho=0=\mathrm{D}_{a} \Theta$ ). These constraints, which are self-consistent (i.e. preserved in time) at the linear perturbative level, guarantee that the 4-acceleration also vanishes to first approximation. When a magnetic field is included there is an additional constraint on the anisotropic pressure of the field. In particular, the system (11.1.1) reads [142,170]

$$
\begin{aligned}
\mathrm{D}^{b} E_{a b} & =\frac{1}{3} \mathrm{D}_{a} \rho+\frac{1}{6} \mathrm{D}_{a} B^{2}-\frac{1}{2} \mathrm{D}^{b} \Pi_{a b}=0, \\
\mathrm{D}^{b} H_{a b} & =\rho(1+w) \omega_{a}=0, \\
\mathrm{D}^{b} \sigma_{a b} & =\frac{2}{3} \mathrm{D}_{a} \Theta+\operatorname{curl} \omega_{a}=0, \\
\mathrm{D}^{b} \Pi_{a b} & =\varepsilon_{a b c} B^{b} \operatorname{curl} B^{c}-\frac{1}{6} \mathrm{D}_{a} B^{2}=0 .
\end{aligned}
$$

When studying the magnetic effects on cosmological gravitational waves, one needs to take into account the anisotropic nature of the $B$-field. It is therefore not appropriate to assume a fully random $B$-field, which simply acts as an additional source of energy density. Thus, following [68] and also [142,170], we will treat both the energy density and the anisotropic pressure of the field as first order perturbations 13 This approach differs from the perturbative scheme adopted for the scalar and the vector modes, where there was a weak $B$-field in the background. Nevertheless, adopting the scheme of [68] simplifies the mathematics of the gravito-magnetic interaction without compromising the physics.

We assume a spatially flat FRW background. Then one can isolate the pure-tensor perturbations by adding to the previously mentioned perfect-fluid constraints the following linear conditions [142,170]

$$
\mathrm{D}_{a} B^{2}=0=\varepsilon_{a b c} B^{b} \operatorname{curl} B^{c} .
$$

In other words, both magnetic energy-density gradients and the Lorentz force vanish at all times. These guarantee that there is no magnetic contribution to the 4-acceleration and subsequently that the field does not trigger any vorticity, density or expansion perturbations. The consistency of constraints (11.1.6) is straightforward to show. In particular, we find that

$$
\left(\mathrm{D}_{a} B^{2}\right)^{\cdot}=-5 H\left(\mathrm{D}_{a} B^{2}\right) \quad \text { and } \quad\left(\varepsilon_{a b c} B^{b} \operatorname{curl} B^{c}\right)^{\cdot}=-5 H \varepsilon_{a b c} B^{b} \operatorname{curl} B^{c} \text {, }
$$

to lowest order. This means that the constraints (11.1.6) continue to hold after they are initially imposed. Then, the only remaining nontrivial linear constraints are

$$
H_{a b}=\operatorname{curl} \sigma_{a b} \quad \text { and } \quad \mathcal{R}_{\langle a b\rangle}=-H \sigma_{a b}+E_{a b}+\frac{1}{2} \Pi_{a b},
$$

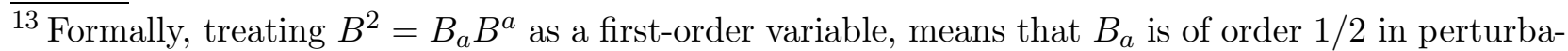
tive terms. Then, because $\mathrm{D}_{a} B^{2}$ is of order 1 and $\mathrm{D}_{a} B^{2}=2 B^{b} \mathrm{D}_{a} B_{b}$, consistency demands that the projected magnetic gradients are also half-order perturbations.
} 
where curl $\sigma_{a b}=\varepsilon_{c d\langle a} \mathrm{D}^{c} \sigma_{b\rangle}{ }^{d}$ (see Eqs. (2.3.7) and (4.3.6) in $\S 2.3$ and $\S 4.3$ respectively). Note that, according to (11.1.8 $\mathrm{b}$ ), the linear conditions $\mathrm{D}^{b} E_{a b}=0=\mathrm{D}^{b} \sigma_{a b}=\mathrm{D}^{b} \Pi_{a b}$ guarantee that $\mathrm{D}^{b} \mathcal{R}_{\langle a b\rangle}=0$ as well.

\subsection{Covariant gravitational-wave energy density}

The energy density of gravitational radiation is determined by the pure tensor, namely the transverse traceless part $\left(h_{\alpha \beta}^{T T}\right)$ of the metric perturbation, according to (e.g. see [171])

$$
\rho_{G W}=\frac{\left(h_{\alpha \beta}^{T T}\right)^{\prime}\left(h_{T T}^{\alpha \beta}\right)^{\prime}}{2 a^{2}}
$$

where a prime indicates differentiation with respect to conformal time (recall that $c=1=8 \pi G$ throughout this review). In a comoving frame, with $u^{a}=\delta_{0}{ }^{a} u^{0}$, we have $\sigma^{2}=\sigma_{a b} \sigma^{a b} / 2=$ $\sigma_{\alpha \beta} \sigma^{\alpha \beta} / 2$, with the transverse part of the shear components given by [159,172]

$$
\sigma_{\alpha \beta}=a\left(h_{\alpha \beta}^{T T}\right)^{\prime} \quad \text { and } \quad \sigma^{\alpha \beta}=a^{-3}\left(h_{T T}^{\alpha \beta}\right)^{\prime} .
$$

Solving these relations for the metric perturbations and then substituting the results into Eq. (11.2.1), we arrive at [142]

$$
\rho_{G W}=\sigma^{2},
$$

which provides a simple covariant expression for the energy density of gravitational-wave distortions.

\subsection{Evolution equations}

In an FRW spacetime the Weyl tensor vanishes identically, which means that $E_{a b}$ and $H_{a b}$ provide a covariant and gauge-invariant description of perturbations in the free-gravitational field. Once the pure tensor modes have been isolated, we can proceed to linearize the propagation equations (4.2.5), (4.2.6) of $\S$ 4.2. On a Friedmann background with a single perfect fluid, the latter reduce to [142,170]

$$
\begin{aligned}
& \dot{E}_{a b}=-3 H E_{a b}-\frac{1}{2} \rho(1+w) \sigma_{a b}+\operatorname{curl} H_{a b}-\frac{1}{2} \dot{\Pi}_{a b}-\frac{1}{2} H \Pi_{a b}, \\
& \dot{H}_{a b}=-3 H H_{a b}-\operatorname{curl} E_{a b}+\frac{1}{2} \operatorname{curl} \Pi_{a b} .
\end{aligned}
$$

Since the magnetic part of the Weyl tensor satisfies the constraint (11.1.8 a), the linear evolution of $H_{a b}$ is determined by the shear evolution (2.3.3)

$$
\dot{\sigma}_{a b}=-2 H \sigma_{a b}-E_{a b}+\frac{1}{2} \Pi_{a b}
$$


Furthermore, on using the commutation law between the orthogonally projected gradients of spacelike tensors, constraint (11.1.8 a) leads to the auxiliary relation $\operatorname{curl} H_{a b}=-\mathrm{D}^{2} \sigma_{a b}$, and Eq. (11.3.1) becomes

$$
\dot{E}_{a b}=-3 H E_{a b}-\frac{1}{2} \rho(1+w) \sigma_{a b}-\frac{1}{2} \dot{\Pi}_{a b}-\frac{1}{2} H \Pi_{a b}-\mathrm{D}^{2} \sigma_{a b} .
$$

This, together with Eq. (11.3.3) and the linearized part of (5.2.2), namely

$$
\dot{\Pi}_{a b}=-4 H \Pi_{a b},
$$

describe the linear evolution of gravitational waves in almost-FRW universes containing a single highly conductive barotropic fluid and a magnetic field.

We can describe this evolution in terms of the shear as follows. Taking the time derivative of (11.3.3), using Eqs. (11.3.4), (11.3.5), the background Raychaudhuri and Friedmann equations, and keeping only linear order terms, we arrive at the following wave equation for the gravitationally induced shear [170]

$$
\ddot{\sigma}_{a b}-\mathrm{D}^{2} \sigma_{a b}=-5 H \dot{\sigma}_{a b}-\frac{1}{2} \rho(1-3 w) \sigma_{a b}-2 H \Pi_{a b} .
$$

This is no longer coupled to the electric Weyl tensor, and together with Eq. (11.3.5), describes the propagation of gravitational waves. The magnetic anisotropic stress can act as a source for gravitational waves, and it is possible to place strong constraints on magnetic fields via this generation of gravitational waves [171/173]. Also, the anisotropic stress of the $B$-field slows the decay of shear [174].

Following (11.3.6), the solution of Eqs. (11.3.5) and (11.3.6) depends critically on the equation of state of the matter component, and in particular on whether $w=1 / 3$ or not. In the matter dominated era and on superhorizon scales, we find

$$
\sigma=P_{1} t^{-1 / 3}+P_{2} t^{-2}+Q t^{-5 / 3}
$$

where $\dot{P}_{1}=0=\dot{P}_{2}=\dot{Q}$. The $P$-modes are the usual Bianchi $I$ type solutions, while the $Q$-mode carries the effect of the $B$-field. The latter mode means that, although the magnetic presence does not alter the standard evolution rate of the shear, it can affect its magnitude (in a rather involved way - see [142]). Note that in deriving the above we have also introduced the familiar tensor harmonics $\mathcal{Q}_{a b}^{(k)}$, with $\mathcal{Q}_{a b}^{(k)}=\mathcal{Q}_{\langle a b\rangle}^{(k)}, \dot{\mathcal{Q}}_{a b}^{(k)}=0=\mathrm{D}^{b} \mathcal{Q}_{a b}^{(k)}$ and $\mathrm{D}^{2} \mathcal{Q}_{a b}^{(k)}=$ $-(k / a)^{2} \mathcal{Q}_{a b}^{(k)}$. These were used to decompose $\sigma_{a b}$ and $\Pi_{a b}$ according to $\sigma_{a b}=\sum_{k} \sigma_{(k)} \mathcal{Q}_{a b}^{(k)}$ and $\Pi_{a b}=\sum_{k} \Pi_{(k)} \mathcal{Q}_{a b}^{(k)}$ respectively (with $\left.\mathrm{D}_{a} \sigma^{(k)}=0=\mathrm{D}_{a} \Pi^{(k)}\right)$.

\subsection{The zero-eigenvalue issue}

The evolution equation of trace-free anisotropic stresses during the radiation era presents a particular mathematical problem, when linearised around an FRW background, because of 
the zero eigenvalue issue (see [65] and references there in). In particular, following Eq. (6.1.6), it appears that the Alfvén speed and therefore the ratio $B^{2} / \rho$ remain constant as long as $w=1 / 3$. This is a critical case, however, and a closer analysis leads to $B^{2} / \rho \propto(\log t)^{-1}$ because of second-order pressure effects. When these are taken into account, we find that [174]

$$
\sigma_{a b}=P_{a b} t^{-3 / 2}+Q_{a b}(t \log t)^{-1},
$$

on superhorizon scales. The magnetized mode falls off slowly due to the anisotropic pressures, with $\sigma_{a b} \propto(t \log t)^{-1}$, and there is an attractor with $\sigma / H \rightarrow B^{2} / \rho$ and $\Pi_{a b} \rightarrow 2 E_{a b} \propto$ $\left(t^{2} \log t\right)^{-1}$ when the dynamics of the radiation era are close to isotropy [65]174]. In this regime the leading-order diagonal scale factors of the spacetime metric during the radiation epoch take the form

$$
a_{i}(t) \propto t^{1 / 2}(\log t)^{q_{i}},
$$

with the $\left\{q_{i}, i=1,2,3\right\}$ constants satisfying the constraint $\sum_{i=1}^{3} q_{i}=0$. In the dust-dominated era the anisotropies fall as power-law in time and the orthogonal scale factors evolve as

$$
a_{i}(t) \propto t^{2 / 3}\left(1+V_{i} t^{-s_{i}}\right),
$$

where the $\left\{V_{i}, i=1,2,3\right\}$ and the $\left\{s_{i}, i=1,2,3\right\}$ are sets of constants with $\sum_{i=1}^{3} s_{i}=0$. Since the observed microwave background temperature anisotropy is determined by the value of $\sigma / H$ at recombination, and $\sigma / H \propto B^{2} / \rho_{m} \propto t^{-2 / 3}$ after the epoch of matter-radiation equality, we obtain a strong observational bound on the present-day magnetic field strength from the large-angular scale anisotropy of the microwave background at last scattering, when $z \sim 1100$, [66]:

$$
|B|<4.0 \times 10^{-9} \Omega_{0}^{1 / 2} G .
$$

An investigation of the behaviour of Yang-Mills fields in the early universe reveals that chaotic behaviour is possible in the evolution of the scale factors with time, no matter how weak the Yang-Mills field, and how close the expansion is to isotropy [175[176], if there is no perfect fluid present in addition to the magnetic field. However, despite the close relationship between the form of Yang-Mills fields and magnetic fields, the slow logarithmic decay of $\sigma / H \propto(\log t)^{-1}$ of the shear distortion with time characteristic of magnetic fields and radiation does not occur if an isotropic blackbody radiation fluid is added to the Yang-Mills fields [177], because of the different time-dependence of their pressures $\left(\Pi_{a b} / \rho_{Y M}\right.$ is no longer constant), which hastens their decay, and the shear falls off in Bianchi $I$ universes at the same rate as it does when anisotropic stresses are absent, with $\sigma / H \propto t^{-1 / 2}$. This means that the microwave background anisotropy provides a rather weak bound on the energy-density in Yang-Mills fields today of $\Omega_{Y M 0}<0.11 \Omega_{\gamma 0}$, where $\Omega_{\gamma 0}$ is the present density of blackbody fluids in units of the critical density. By contrast, the slow decay of the shear in the presence of magnetic stresses led to a far stronger bound on the magnetic field density today, with $\Omega_{m a g 0} \lesssim 10^{-5} \Omega_{\gamma 0}$. 


\section{Summary}

Magnetic fields are detected everywhere when the appropriate observations are possible. The origin of cosmic magnetism is still an open issue but the presence of large-scale $B$-fields with similar $\mu \mathrm{G}$-order strengths in galaxies, galaxy clusters, and high-redshift protogalactic structures may suggest a common (primordial) origin for them. Large-scale, micro-Gauss fields can significantly affect the evolution of the universe and are extremely important for astrophysics (see [12] for a cross-disciplinary review of the subject). The idea of primordial magnetism is appealing because it can potentially explain all the large-scale fields seen in the universe today, especially those found in remote protogalaxies. As a result, the literature contains many studies examining the role and the implications of magnetic fields for cosmology.

In this work we have employed covariant techniques to analyse the effects of magnetic fields on the evolution of inhomogeneous relativistic cosmologies. After a brief introduction to nonlinear cosmological electrodynamics and magnetohydrodynamics, we investigated weakly magnetized almost-FRW universes. Within the standard ideal MHD limits, we found that linear inhomogeneities in the magnetic energy density grow in step with those in the matter throughout the hot big-bang evolution of the universe. This close relation between the magnetic and the fluid density gradients is a key property of weakly-magnetized FRW cosmologies.

We found that during the radiation era and on large scales the magnetic pressure inhibits the growth of density perturbations by an amount proportional to the field's relative strength. On sub-horizon lengths the extra magnetic pressure was shown to trigger magnetosonic waves with a reduced amplitude and an increased frequency, relative to the magnetic-free case. Moreover, the magnetic presence means that small-scale density perturbations no longer oscillate around a zero average value but have a finite residual average that depends on the initial conditions. After equality, the $B$-field is effectively the only source of pressure support. This reduces the growth rate of matter condensations in a way exactly analogous to that observed on super-Hubble scales during the radiation era. The same magnetic pressure can also stabilize against gravitational collapse by bringing the Jeans length up to the size of a galaxy cluster. Looking at models with a vacuum equation of state for the matter, we found that the outcome depends on the effective equation of state of the medium. In particular, the comoving density gradients remain constant as long as the barotropic $p=-\rho$ condition holds. When dealing with non-barotropic minimally coupled scalar fields, on the other hand, all three types of density inhomogeneities suffer exponential decay.

Defining as isocurvature large-scale perturbations that maintain the zero 3-curvature requirement at all times, we found that they contain decaying modes only. Given that, typically, the term 'isocurvature' refers to fluctuations with zero initial total energy-density perturbation, we have explained how our equations can be used to study this type of perturbation mode. By treating the highly conductive medium as a two-component system we found that the effective entropy perturbation caused by the different dynamical evolution of the two species is either constant or zero. The evolution of vector perturbations in the presence of magnetic fields, showed that the latter increase the amount of residual vorticity. Thus, universes containing 
large-scale inhomogeneous $B$-fields would rotate more than their magnetic-free counterparts. Finally, we investigated the implications of the field for the evolution of gravitational-wave perturbations.

Acknowledgements: CT wishes to thank the Centre for Mathematical Sciences at Cambridge University and the Institute of Cosmology and Gravitation at Portsmouth University for their hospitality on a number of occasions while this work was in progress and PPARC for support in Cambridge. The work of RM was partly supported by PPARC.

\section{A Commutation laws}

According to definition (2.1.2 $\mathrm{a})$, the orthogonally projected covariant derivative operator satisfies the condition $\mathrm{D}_{a} h_{b c}=0$. This means that we can use $h_{a b}$ to raise and lower indices in equations acted upon by the aforementioned operator. Following Frobenius' theorem, however, rotating spaces do not possess integrable 3-D submanifolds (e.g. see [140,141]). Therefore, the $\mathrm{D}_{a}$-operator cannot be used as a standard 3-D derivative in such spaces and it does not always satisfy the usual commutation laws (see below and also [149]).

\section{A.1 Orthogonally projected gradients}

When acting on a scalar quantity the orthogonally projected covariant derivative operators commute according to

$$
\mathrm{D}_{[a} \mathrm{D}_{b]} f=-\omega_{a b} \dot{f} .
$$

The above is a purely relativistic result and underlines the different behaviour of rotating spacetimes within Einstein's theory. Similarly, the commutation law for the orthogonally projected derivatives of spacelike vectors reads

$$
\mathrm{D}_{[a} \mathrm{D}_{b]} v_{c}=-\omega_{a b} \dot{v}_{\langle c\rangle}+\frac{1}{2} \mathcal{R}_{d c b a} v^{d}
$$

where $v_{a} u^{a}=0$ and $\mathcal{R}_{a b c d}$ represents the Riemann tensor of the observer's local rest-space. Finally, when dealing with orthogonally projected tensors, we have

$$
\mathrm{D}_{[a} \mathrm{D}_{b]} S_{c d}=-\omega_{a b} h_{c}{ }^{e} h_{d}^{f} \dot{S}_{e f}+\frac{1}{2}\left(\mathcal{R}_{e c b a} S_{d}^{e}+\mathcal{R}_{e d b a} S_{c}{ }^{e}\right)
$$

with $S_{a b} u^{a}=0=S_{a b} u^{b}$. Note that in the absence of rotation, $\mathcal{R}_{a b c d}$ is the Riemann tensor of the (integrable) 3-D hypersurfaces orthogonal to the $u_{a}$-congruence For details on the definition, the symmetries and the key equations involving $\mathcal{R}_{a b c d}$, the reader is referred to $\S 4.3$. We also note that the above equations are fully nonlinear and hold at all perturbative levels. 


\section{A.2 Time derivatives}

In general relativity, time derivatives do not generally commute with their spacelike counterparts. For scalars, in particular, we have

$$
\mathrm{D}_{a} \dot{f}-h_{a}{ }^{b}\left(\mathrm{D}_{b} f\right)^{\cdot}=-\dot{f} A_{a}+\frac{1}{3} \Theta \mathrm{D}_{a} f+\mathrm{D}_{b} f\left(\sigma^{b}{ }_{a}+\omega^{b}{ }_{a}\right)
$$

at all perturbative levels. Assuming an FRW background, we find that the orthogonally projected gradient and the time derivative of the first-order vector $v_{a}$ commute as

$$
a \mathrm{D}_{a} \dot{v}_{b}=\left(a \mathrm{D}_{a} v_{b}\right)^{\cdot}
$$

to linear order. Similarly, when dealing with first-order spacelike tensors, we have the following linear commutation law

$$
a \mathrm{D}_{a} \dot{S}_{b c}=\left(a \mathrm{D}_{a} S_{b c}\right)^{\cdot}
$$

\section{B Notation}

\section{- Spacetime Geometry}

Line element: $\mathrm{d} s^{2}=g_{a b} \mathrm{~d} x^{a} \mathrm{~d} x^{b}=-\mathrm{d} \tau^{2}$, with $c=1$.

4-velocity: $u^{a}=\mathrm{d} x^{a} / \mathrm{d} \tau, 3-\mathrm{D}$ projection tensor: $h_{a b}=g_{a b}+u_{a} u_{b}$.

4-D permutation tensor: $\eta_{a b c d}, 3-\mathrm{D}$ permutation tensor: $\varepsilon_{a b c}=\eta_{a b c d} u^{d}$.

Covariant derivative: $\nabla_{b} T_{a}=\partial T_{a} / \partial x^{b}-\Gamma_{a b}^{c} T_{c}$.

Time derivative: $\dot{T}_{a}=u^{b} \nabla_{b} T_{a}, 3-\mathrm{D}$ covariant derivative: $\mathrm{D}_{b} T_{a}=h_{b}{ }^{d} h_{a}{ }^{c} \nabla_{d} T_{c}$.

Riemann tensor: $R_{a b c d}$, Ricci tensor: $R_{a b}=R_{a c b}^{c}$, Ricci scalar: $R=R_{a}^{a}$.

3-Riemann tensor: $\mathcal{R}_{a b c d}$, 3-Ricci tensor: $\mathcal{R}_{a b}=\mathcal{R}^{c}{ }_{a c b}$, 3-Ricci scalar: $\mathcal{R}=\mathcal{R}^{a}{ }_{a}$.

3-curvature index: $K=0, \pm 1$, with $\mathcal{R}=6 K / a^{2}$ (in FRW models).

Weyl Tensor: $C_{a b c d}$, electric Weyl: $E_{a b}=C_{a c b d} u^{c} u^{d}$, magnetic Weyl: $H_{a b}=\varepsilon_{a}{ }^{c d} C_{c d b e} u^{e} / 2$.

- Kinematics

Expansion scalar: $\Theta=\nabla^{a} u_{a}=\mathrm{D}^{a} u_{a}$, scale factor: $a$, with $\dot{a} / a=\Theta / 3$.

Vorticity tensor: $\omega_{a b}=\mathrm{D}_{[b} u_{a]}$, vorticity vector: $\omega_{a}=\varepsilon_{a b c} \omega^{b c} / 2$.

Shear tensor: $\sigma_{a b}=\mathrm{D}_{\langle b} u_{b\rangle}=\mathrm{D}_{(b} u_{b)}-\left(\mathrm{D}^{c} u_{c}\right) h_{a b} / 3$, 4-acceleration: $A_{a}=u^{b} \nabla_{b} u_{a}$.

Hubble parameter: $H=\dot{a} / a$ (in FRW models).

- Matter Fields

Field equations: $R_{a b}-(R / 2) g_{a b}=T_{a b}$, with $\kappa=8 \pi G=1$.

Matter energy-momentum tensor: $T_{a b}=\rho u_{a} u_{b}+p h_{a b}+2 u_{(a} q_{b)}+\pi_{a b}$.

Matter density: $\rho=T_{a b} u^{a} u^{b}$, isotropic pressure: $p=T_{a b} h^{a b} / 3$.

Barotropic index: $w=p / \rho$, adiabatic sound speed: $c_{\mathrm{s}}^{2}=\partial p / \partial \rho$.

Energy flux: $q_{a}=h_{a}{ }^{b} T_{b c} u^{c}$, anisotropic pressure: $\pi_{a b}=T_{\langle a b\rangle}=T_{(a b)}-(T / 3) h_{a b}$.

- Electromagnetism

Electromagnetic tensor: $F_{a b}$, magnetic field: $B_{a}=\varepsilon_{a b c} F^{b c} / 2$, electric field: $E_{a}=F_{a b} u^{b}$. Magnetic energy density: $B^{2} / 2$, magnetic isotropic pressure: $B^{2} / 6$. 
Magnetic anisotropic pressure: $\Pi_{a b}=-B_{\langle a} B_{b\rangle}=\left(B^{2} / 3\right) h_{a b}-B_{a} B_{b}$.

Alfvén speed: $c_{\mathrm{a}}^{2}=B^{2} /\left(\rho+p+B^{2}\right)$.

Electric 4-current: $J_{a}$, electric 3-current: $\mathcal{J}_{a}=J_{\langle a\rangle}=h_{a}{ }^{b} J_{b}$.

Charge density: $\rho_{e}=-J_{a} u^{a}$, electrical conductivity: $\varsigma$.

- Perturbations

Matter density gradients: $\Delta_{a}=(a / \rho) \mathrm{D}_{a} \rho$, with $\Delta_{a b}=a \mathrm{D}_{b} \Delta_{a}$ and $\Delta=\Delta^{a}{ }_{a}$.

Matter vortices: $\mathcal{W}_{a b}=\Delta_{[a b]}$, with $\mathcal{W}_{a}=\varepsilon_{a b c} \mathcal{W}^{b c} / 2$.

Volume expansion gradients: $\mathcal{Z}_{a}=a \mathrm{D}_{a} \Theta$, with $\mathcal{Z}_{a b}=a \mathrm{D}_{b} \mathcal{Z}_{a}$ and $\mathcal{Z}=\mathcal{Z}^{a}{ }_{a}$.

Magnetic density gradients: $\mathcal{B}_{a}=\left(a / B^{2}\right) \mathrm{D}_{b} B^{2}$ and $\mathcal{B}_{a b}=a \mathrm{D}_{b} \mathcal{B}_{a}$, with $\mathcal{B}=\mathcal{B}^{a}{ }_{a}$.

Effective entropy perturbations: $\mathcal{E}_{a}, \mathcal{S}_{a}^{(i j)}$, with $\mathcal{S}_{a}^{(i j)}=-\mathcal{S}_{a}^{(j i)}$.

\section{References}

[1] U. Klein, R. Wielebinski and H.W. Morsi, Astron. Astrophys. 190, 41 (1988).

[2] P.P. Kronberg, J.J. Perry and E.L.H. Zukowski, Astrophys. J. 387, 528 (1992).

[3] A.M. Wolfe, K.M. Lanzetta and A.L. Oren, Astrophys. J. 388, 17 (1992).

[4] P.P. Kronberg, Rep. Prog. Phys. 57, 325 (1994).

[5] R. Beck, A. Brandenburg, D. Moss, A.A. Shukurov and D. Sokoloff, Ann. Rev, Astron. Astrophys. 34, 155 (1996).

[6] J.P. Vallée, New Astron. Rev. 48, 763 (2004).

[7] C.L. Carilli and G.B. Taylor, Ann. Rev. Astron. Astrophys. 40, 391 (2004).

[8] K. Enqvist, Int. J. Mod. Phys. D 7, 331 (1998).

[9] D. Grasso and H. Rubinstein, Phys. Rep. 348, 163 (2001).

[10] L.M. Widrow, Rev. Mod. Phys. 74, 775 (2002).

[11] M. Giovannini, in 7th Paris Cosmology Colloquium on High Energy Astrophysics for and from Space, Eds. N. Sanchez and H. de Vega, (2002) (hep-ph/0208152).

[12] M. Giovannini, Int. J. Mod. Phys. D 13, 391 (2004).

[13] E.N. Parker, Cosmical Magnetic Fields (Oxford, Clarendon 1979).

[14] Y.B. Zeldovich, A.A. Ruzmaikin and D.D. Sokoloff, Magnetic Fields in Astrophysics (New York, Gordon and Breach 1983).

[15] F. Krause, in The Cosmic Dynamo, Eds. F. Krause, K.-H. Rädler and G. Rüdiger (Kluwer, Dordrecht, 1993) p. 487.

[16] L. Mestel, Stellar Magnetism (Oxford, Oxford University Press 1999).

[17] A. Brandenburg and K. Subramanian, Phys. Rep. 417, 1 (2005). 
[18] R.M. Kulsrud, in Galactic and Extragalactic Magnetic Fields, Eds. R. Beck, P.P. Kronberg, and R. Wielebinski (Reidel, Dordrecht, 1990).

[19] R.M. Kulsrud and S.W. Anderson, Astrophys. J. 396, 606 (1992).

[20] A.-C. Davis, M. Lilley and O. Törnkvist, Phys. Rev. D 60, 021301 (1999).

[21] E.R. Harrison, Mon. Not. R. Astron. Soc. 147, 279 (1970).

[22] R.E. Pudritz and J. Silk, Astrophys. J. 342, 650 (1989).

[23] R.M. Kulsrud, R. Cen, J.P. Ostriker and D. Ryu, Astrophys. J. 480, 481 (1997).

[24] T. Vachaspati, Phys. Lett. B 265, 258 (1991).

[25] K. Enqvist and P. Olesen, Phys. Lett. B 319, 178 (1993).

[26] M. Gasperini, M. Giovannini and G. Veneziano, Phys. Rev. Lett. 75, 3796 (1995).

[27] A. Brandenburg, K. Enqvist and P. Olesen, Phys. Rev. D 54, 1291 (1996).

[28] G. Sigl, K. Jedamzik and A.V. Olinto, Phys. Rev. D 55, 4582 (1997).

[29] A.-C. Davis and K. Dimopoulos, Phys. Rev. D 55, 7398 (1997).

[30] E.A. Calzetta, A. Kandus and F.D Mazzitelli, Phys. Rev. D 57, 7139 (1998).

[31] A. Kandus, E.A. Calzetta, F.D Mazzitelli and C.E.M. Wagner, Phys. Lett. B 472, 287 (2000).

[32] M. Gasperini, Phys. Rev. D 63, 047301 (2001).

[33] E.A. Calzetta and A. Kandus, Phys. Rev. D 65, 063004 (2002).

[34] R. Durrer and C. Caprini, JCAP 0311, 010 (2003).

[35] M.R. Garousi, M. Sami and S. Tsujikava, Phys. Lett. B 606, 1 (2005).

[36] K. Takahashi, K. Ichiki, H. Ohno and H. Hanayama, Phys. Rev. Lett. 95, 121301 (2005).

[37] A.-C. Davis and K. Dimopoulos, Phys. Rev. D 72, 043517 (2005).

[38] K.E. Kunze, Phys. Lett. B 623, 1 (2005).

[39] M. Gasperini, in The Origin and Evolution of Cosmic Magnetism, Eds. R. Beck, G. Brunetti, L. Feretti and B. Gaensler, Astron. Nachr 327, 399 (2006).

[40] S. Matarrese, S. Mollerach, A. Notari and A. Riotto, Phys. Rev. D, 71, 043502 (2005).

[41] R. Gopal and S. Sethi, Mon. Not. Roy. Astron. Soc., 363, 529 (2005).

[42] E. R. Siegel and J. N. Fry, preprint: astro-ph/0604526.

[43] T. Kobayashi, R. Maartens, T. Shiromizu and K. Takahashi, Phys. Rev. D 75, 103501 (2007).

[44] K. Ichiki, K. Takahashi, N. Sugiyama, H. Hanayama and H. Ohno, preprint: astro-ph/0701329.

[45] K. Subramanian and J.D. Barrow, Phys. Rev. D 58, 083502 (1998). 
[46] K. Subramanian and J.D. Barrow, Phys. Rev. Lett. 81, 3575 (1998).

[47] K. Subramanian, T.R. Seshadri and J.D. Barrow, Mon. Not. R. Astron. Soc. 344, L31 (2003).

[48] M.S. Turner and L. Widrow, Phys. Rev. D 37, 2743 (1988).

[49] B. Ratra, Astrophys. J. 391, L1 (1992).

[50] M. Giovannini, Phys. Rev. D 62, 123505 (2000).

[51] B.A. Bassett, G. Pollifrone, S. Tsujikawa and F. Viniegra, Phys. Rev. D 63, 103515 (2001).

[52] A.L. Maroto, Phys. Rev D 64, 083006 (2001).

[53] K. Dimopoulos, T. Prokopec, O. Törnkvist and A.-C. Davis, Phys. Rev. D 65, 063505 (2002).

[54] C.G. Tsagas, P.K.S. Dunsby and M. Marklund, Phys. Lett. B 561, 17 (2003).

[55] A. Ashoorioon and R.B. Mann, Phys. Rev. D 71, 103509 (2005).

[56] C.G. Tsagas and A. Kandus, Phys. Rev. D 71, 103509 (2005).

[57] C.G. Tsagas, Phys. Rev. D 72, 123509 (2005).

[58] B.A. Bassett, S. Tsujikawa and D. Wands, Pev. Mod. Phys. 78, 537 (2006).

[59] D. Grasso and H.R. Rubinstein, Phys. Lett. B 379, 79 (1996).

[60] B. Cheng, A.V. Olinto, D.N. Schramm and J.W. Truran, Phys. Rev. D 54, 4714 (1997).

[61] Y.B. Zeldovich, Sov. Astron. 13, 608 (1970).

[62] Y.B. Zeldovich and I.D. Novikov, Relativistic Astrophysics: Volume II (Chicago, University of Chicago Press 1974).

[63] J. Adams, U.H. Danielson, D. Grasso and H.R. Rubinstein, Phys. Lett. B 388, 253 (1996).

[64] A. Kosowsky and A. Loeb, Astrophys. J. 469, 1 (1996).

[65] J.D. Barrow, Phys. Rev. D 55, 7451 (1997).

[66] J.D. Barrow, P.G. Ferreira and J. Silk, Phys. Rev. Lett. 78, 3610 (1997).

[67] R. Durrer, T. Kahniasvili and A. Yates, Phys. Rev. D 58, 123004 (1998).

[68] R. Durrer, P.G. Ferreira and T. Kahniashvili, Phys. Rev. D 61, 043001 (2000).

[69] L. Pogosian, T. Vachaspati and S. Winitzki, Phys. Rev. D 65, 083502 (2002).

[70] C.A. Clarkson, A.A. Coley, R. Maartens and C.G. Tsagas, Class. Quantum Grav. 20, 1519 (2003).

[71] C. Caprini, R. Durrer and T. Kahniashvili, Phys. Rev. D 69, 063006 (2004).

[72] A. Lewis, Phys. Rev. D 70, 043011 (2004).

[73] M. Giovannini, Phys. Rev. D 70, 123507 (2004). 
[74] M. Giovannini, Phys. Rev. D 71, 021301 (2005).

[75] M. Giovannini, Class. Quantum Grav. 23, R1 (2006).

[76] M. Giovannini, Phys. Rev D 73, 101302 (2006).

[77] K. Subramanian, in The Origin and Evolution of Cosmic Magnetism, Eds. R. Beck, G. Brunetti, L. Feretti and B. Gaensler, Astron. Nachr 327, 403 (2006).

[78] D. Yamazaki, K. Ichiki, T. Kajino and G.J. Mathews, Astrophys. J. 646, 719 (2006).

[79] R. Durrer, New Astron. Rev. 51, 275 (2007).

[80] T. Kahniashvili and B. Ratra, Phys. Rev. D 75, 023002 (2007).

[81] K.S. Thorne, Astrophys. J. 148, 51 (1967).

[82] K. Jacobs, Astrophys. J. 155, 379 (1969).

[83] C.B. Collins, Commun. Math. Phys. 27, 37 (1972).

[84] V.G. LeBlanc, D. Kerr and J. Wainwright, Class. Quantum Grav. 12, 513 (1995).

[85] V.G. LeBlanc, Class. Quantum Grav. 14, 2281 (1997).

[86] M. Weaver, Class. Quantum Grav. 17, 421 (2000).

[87] A. Pradhan and O.P. Pandey, Int. J. Mod. Phys. D 12, 1299 (2003).

[88] J.T. Horwood and J. Wainwright, Gen. Rel. Grav. 36, 799 (2004).

[89] E.J. King and P. Coles, Class. Quantum Grav. 24, 2061 (2007).

[90] T.V. Ruzmaikina and A.A. Ruzmaikin, Sov. Astron. 14, 963 (1971).

[91] I. Wasserman, Astrophys. J. 224, 337 (1978).

[92] P.J.E. Peebles, The Large-Scale Structure of the Universe (Princeton, Princeton University Press 1980).

[93] E. Kim, A.V. Olinto and R. Rosner, Astrophys. J. 468, 28 (1996).

[94] E. Battaner and E. Florido, Astron. Nachr. 328, 92 (2007).

[95] L. Vlahos, C.G. Tsagas and D. Papadopoulos, Astrophys. J. L9 629, (2005).

[96] A.G. Fennelly, Phys. Rev. Lett. 55, 955 (1980).

[97] D. Papadopoulos and F.P. Esposito, Astrophys. J. 257, 10 (1982).

[98] E. Battaner, E. Florido and J. Jimenez-Vicénte, Astron. Astrophys. 326, 13 (1997).

[99] C.G. Tsagas and J.D. Barrow, Class. Quantum Grav. 14, 2539 (1997).

[100] C.G. Tsagas and J.D. Barrow, Class. Quantum Grav. 15, 3523 (1998).

[101] C.G. Tsagas and R. Maartens, Phys. Rev. D 61, 083519 (2000). 
[102] C.G. Tsagas and R. Maartens, Class. Quantum Grav. 17, 2215 (2000).

[103] S. Hobbs and P.K.S. Dunsby, Phys. Rev. D 62, 124007 (2000).

[104] M. Marklund, P.K.S. Dunsby, G. Betschart, M. Servin and C.G. Tsagas, Class. Quantum Grav. 20, 1823 (2003).

[105] J. Moortgat and M. Marklund, Mon. Not. R. Astron. Soc. 369, 1813 (2006).

[106] A. Brandenburg, K. Enqvist and P. Olesen, Phys. Lett. B 392, 395 (1997).

[107] K. Jedamzik, V. Katalinic and A. Olinto, Phys. Rev. D 57, 3264 (1998).

[108] K. Dolag, M. Bartelmann and H. Lesch, Astron. Astrophys. 348, 351 (1999).

[109] M. Bruni, R. Maartens and C.G. Tsagas, Mon. Not. R. Astron. Soc. 338, 785 (2003).

[110] G. Siemieniec-Ozieblo and A. Woszczyna, Astron. Astrophys. 414, 1 (2004).

[111] G. Siemieniec-Ozieblo and Z.A. Golda, Astron. Astrophys. 422, 23 (2004).

[112] R. Banerjee and Jedamzik K., Phys. Rev D 70, 123004 (2004).

[113] K. Dolag, D. Grasso, V. Springel and I. Tkachev, JCAP 0501, 009 (2005).

[114] V.B. Semikoz and D.D. Sokoloff, Int. J. Mod. Phys. D 14, 1839 (2005).

[115] M. Onofri, H. Isliker and L. Vlahos, Phys. Rev. Lett. 96, 151102 (2006).

[116] E. King and P. Coles, Mon. Not. R. Astron. Soc. 365, 1288 (2006).

[117] L. Campanelli, P. Cea and L. Tedesco, Phys. Rev. Lett. 97, 131302 (2006).

[118] O. Heckmann and E. Schücking, Zeits. f. Astrophys. 38, 95 (1955).

[119] A.K. Raychaudhuri, Zeits. f. Astrophys. 43, 161 (1957).

[120] G. Ehlers, Abh. Mainz. Akad. Wiss. Lit. 11, 1 (1961).

[121] G.F.R. Ellis, in General Relativity and Cosmology Ed. R.K. Sachs (New York, Academic 1971) p. 104.

[122] G.F.R Ellis and H. van Elst, in Theoretical and Observational Cosmology, Ed. M. Lachiéze-Rey (Dordrecht, Kluwer 1999) p. 1.

[123] C.G. Tsagas, A. Challinor and R. Maartens, preprint: arXiv:0705.4397.

[124] A.R. King and G.F.R Ellis, Commun. Math. Phys. 31, 209 (1973).

[125] H. Kodama and M. Sasaki, Prog. Theor. Phys. Suppl. 78, 1 (1984).

[126] P.K.S. Dunsby, M. Bruni and G.F.R. Ellis, Astrophys. J. 395, 54 (1992).

[127] M. Giovannini, Class. Quantum Grav. 22, 5243 (2005).

[128] G.F.R. Ellis and C.G. Tsagas, Phys. Rev. D 66, 124015 (2002). 
[129] G.F.R. Ellis, in Cargèse Lectures in Physics vol VI, Ed. E. Schatzmann (New York, Gordon and Breach 1973) p. 1.

[130] C.G. Tsagas, Class. Quantum Grav. 22, 393 (2005).

[131] P.J. Greenberg, Ap. J. 164, 589 (1971).

[132] J.D. Jackson, Classical Electrodynamics (New York, Wiley 1975).

[133] S.W. Hawking and G.F.R. Ellis, The Large Scale Structure of Space-time (Cambridge, Cambridge University Press 1973).

[134] R. Maartens, Phys. Rev. D 55, 463 (1997).

[135] R. Maartens, T. Gebbie and G.F.R. Ellis, Phys. Rev. D 59, 083506 (1998).

[136] L. Bel, C.R. Acad. Sci. Paris 247, 1094 (1958).

[137] R. Penrose, Ann. Phys. 138, 59 (1969).

[138] R. Maartens and B. Bassettt, Class. Quantum Grav. 15, 705 (1998).

[139] N. Dadhich, Gen. Rel. Grav. 32, 1009 (2000).

[140] R.M. Wald, General Relativity (Chicago: University of Chicago Press, 1984).

[141] E. Poisson A Relativist's Toolkit (Cambridge, Cambridge University Press, 2004).

[142] C.G. Tsagas, Class. Quantum Grav. 19, 3709 (2002).

[143] D.R. Matravers and C.G. Tsagas, Phys. Rev. D 62, 103519 (2000).

[144] C.G. Tsagas, Phys. Rev. Lett. 86, 5421 (2001).

[145] F. de Felice, F. Sorge and S. Zilio, Class. Quantum Grav. 22, 47 (2005).

[146] C.G. Tsagas, Class. Quantum Grav. 23, 4323 (2006).

[147] G.F.R Ellis and M. Bruni, Phys. Rev. D 40, 1804 (1989).

[148] G.F.R. Ellis, J. Hwang and M. Bruni, Phys. Rev D 40, 1819 (1989).

[149] G.F.R. Ellis, M. Bruni and J. Hwang, Phys. Rev D 42, 1035 (1990).

[150] T. Buchert, Gen. Rel. Grav. 33, 1381 (2001).

[151] J.D. Barrow, Mon. Not. R. Astron. Soc. 179, 47P (1977).

[152] J.M. Stewart and M. Walker, Proc. R. Soc. A 341, 49 (1974).

[153] M. Bruni, S. Matarrese, S. Mollerach and S. Sonego, Class. Quantum Grav. 14, 2584 (1997).

[154] R. Maartens and J. Triginer, Phys. Rev. D 56, 4640 (1997).

[155] P.J.E. Peebles, The Large-Scale Structure of the Universe (Princeton, Princeton University Press, 1980). 
[156] T. Padmanabhan, Structure Formation in the Universe (Cambridge, Cambridge University Press, 1993).

[157] C.G. Tsagas, in Cosmlogical Crossroads, Eds. S. Cotsakis and E. Papantonopoulos, Lec. Notes Phys. 592, 223 (Berlin, Springer, 2002).

[158] G.F.R. Ellis and M.S. Madsen, Class. Quantum Grav. 8, 667 (1991).

[159] M. Bruni, G.F.R. Ellis and P.K.S. Dunsby, Class. Quantum Grav. 9, 921 (1992).

[160] M.S. Madsen, Class. Quantum Grav. 5, 627 (1988).

[161] J.D. Barrow, in The Very Early Universe, Eds. G. Gibbons, S.W. Hawking and S.T.C. Siklos, (Cambridge, Cambridge University Press, 1983) p. 267.

[162] W. Boucher and G.W. Gibbons, in The Very Early Universe, Eds. G. Gibbons, S.W. Hawking and S.T.C. Siklos, (Cambridge, Cambridge University Press, 1983) p. 273.

[163] A.A. Starobinsky, Sov. Astron. Lett. 9, 302 (1983).

[164] R. Wald, Phys. Rev. D 28, 2118 (1983).

[165] V.F. Mukhanov, H.A. Feldman and B.R. Brandenberger, Phys. Rep. 215, 203 (1992).

[166] M. Giovannini, Class. Quantum Grav. 23, 4991 (2006).

[167] A.R. Liddle and D.H. Lyth, Cosmological Inflation and Large-Scale Structure (Cambridge, Cambridge University Press, 2000).

[168] S.W. Hawking, Astrophys. J. 145, 544 (1966).

[169] A. Challinor, Class. Quantum Grav. 17, 871 (2000).

[170] R. Maartens, C.G. Tsagas and C. Ungarelli, Phys. Rev. D 63, 123507 (2001).

[171] C. Caprini and R. Durrer, Phys. Rev. D 65, 023517 (2002).

[172] S.W. Goode, Phys. Rev. D 39, 2882 (1989).

[173] C. Caprini and R. Durrer, Phys. Rev. D 74, 063521 (2006).

[174] J.D. Barrow and R. Maartens, Phys. Rev. D 59, 043502 (1999).

[175] J.D. Barrow and J. Levin, Phys. Rev. Lett. 80, 656 (1998).

[176] Y. Jin and K-I. Maeda, Phys. Rev. D 71, 064007 (2005).

[177] J.D. Barrow, Y. Jin and K-I Maeda, Phys. Rev. D 72, 103512 (2005). 